International Journal of Modern Physics E (C) World Scientific Publishing Company

\title{
Nuclear Mass Measurements With Radioactive Ion Beams
}

\author{
Michael A. Famiano \\ Physics Department, Western Michigan University \\ 1903 W. Michigan Ave. \\ Kalamazoo, MI 49008-5252 United States of America \\ michael.famiano@wmich.edu
}

Received X XXXXX XXXX

Revised XX XXXXX XXXX

\begin{abstract}
Nuclear masses are the most fundamental of all nuclear properties, yet they can provide a wealth of knowledge, including information on astrophysical sites, constraints on existing theory, and fundamental symmetries. In nearly all applications, it is necessary to measure nuclear masses with very high precision. As mass measurements push to more short-lived and more massive nuclei, the practical constraints on mass measurement techniques become more exacting. Various techniques used to measure nuclear masses, including their advantages and disadvantages are described. Descriptions of some of the world facilities at which the nuclear mass measurements are performed are given, and brief summaries of planned facilities are presented. Future directions are mentioned, and conclusions are presented which provide a possible outlook and emphasis on upcoming plans for nuclear mass measurements at existing facilities, those under construction, and those being planned.
\end{abstract}

Keywords: nuclear masses; accelerator physics; nuclear experiment.

PACS numbers:21.60.-n; 26.30.-k; 29.27.-a

\section{Contents}

1. Introduction . . . . . . . . . . . . . . . . . . . 2

1.1. Applications of Nuclear Masses . . . . . . . . . . . . . . . 2

1.2. Example: Nuclear Masses of Neutron-Rich Nuclei and the Astro-

physical r Process . . . . . . . . . . . . . . . . 3

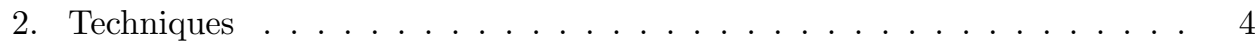

2.1. Penning Traps . . . . . . . . . . . . . . . . 7

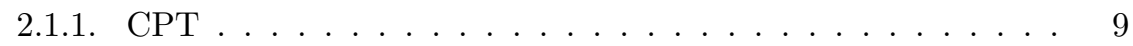

2.1.2. ISOLTRAP . . . . . . . . . . . . . . . . . . . . . . . . . . . . . . .

2.1.3. JYFLTRAP . . . . . . . . . . . . . . . . . . . 11

2.1.4. LEBIT..................... 11

2.1.5. SHIPTRAP .................. . . . 11

2.1.6. TITAN .................... 11

2.2. Other Trap Facilities . . . . . . . . . . . . . . . . . . 12 
2.3. Time-of-Flight Methods . . . . . . . . . . . . . . . . . . . . . . . 12

2.3.1. Rigity-Based TOF Measurements . . . . . . . . . . . . . 13

2.3.2. NSCL . . . . . . . . . . . . . . . . . . 14

2.3.3. Spectromètre à Perte d'Energie du Ganil (SPEG) . . . . . . . 15

2.3.4. Other Facilities . . . . . . . . . . . . . . . . . . . . 17

2.4. Storage Rings Methods . . . . . . . . . . . . . . . . . 17

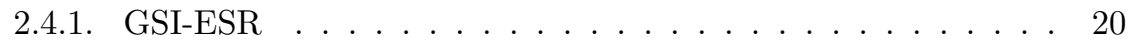

2.4.2. HIRFL-CSRe . . . . . . . . . . . . . . . 20

2.4.3. RIKEN Rare RI-Ring . . . . . . . . . . . . . . . . . 22

2.5. Other Techniques . . . . . . . . . . . . . . . . . . . . . 22

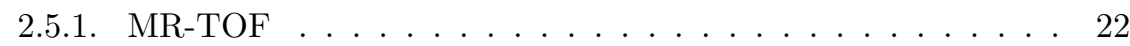

2.5.2. MISTRAL . . . . . . . . . . . . . . 23

3. Mass Measurement Compilations and Evaluations . . . . . . . . . . . . 24

4. Future Work . . . . . . . . . . . . . . . . . . 25

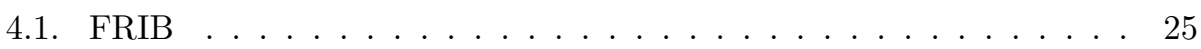

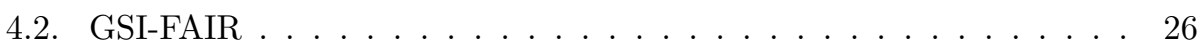

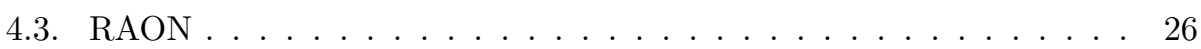

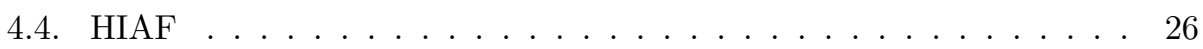

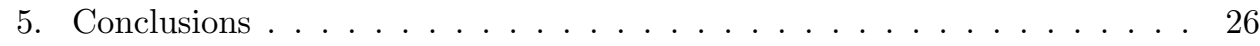

\section{Introduction}

Besides proof of particle stability, perhaps the most fundamental of nuclear properties is nuclear mass. Knowledge of nuclear masses can provide significant insight into other nuclear properties including, but not limited to, particle separation energies, decay rates, shape, and shell model predictions. Because of their intrinsic relationship to a host of other nuclear properties, theoretical predictions and experimental determinations of nuclear masses have been a mainstay of nuclear research virtually since its inception.

Multiple techniques for measuring nuclear mass have evolved over the years, each with characteristic advantages and disadvantages. In many cases, the disadvantages of one particular technique are an advantage of another. Applications of nuclear mass measurements to fundamental nuclear physics and astrophysics will be discussed. Mass measurement techniques will also be discussed along with the facilities at which various methods are used. Finally, future directions in nuclear mass measurements will be presented.

\subsection{Applications of Nuclear Masses}

From an astrophysical standpoint, nuclear masses can provide insight into properties of nucleosynthesis. As an example, the rapid neutron capture process - or $r$ process - is thought to be responsible for production of roughly half of all nuclei heavier than iron ${ }^{1}$ and nearly all of the actinides. Because the r-process proceeds via sequential neutron captures on existing nuclei, ${ }^{A} \mathrm{Z}+\mathrm{n} \rightarrow{ }^{A+1} \mathrm{Z}+\gamma$, the path of 
the r-process is strongly related to the mass of r-process progenitor nuclei. The classical $r$ process path roughly follows a line of constant neutron separation energy, $S_{n}$, where the neutron separation energy is defined to be the amount of energy necessary to remove a neutron from a nucleus, $S_{n} \equiv(M(Z, A)-M(Z, A-1)) c^{2}$. Knowledge of neutron separation energies is important in understanding which nuclei are of particular importance for the r-process. In thermal equilibrium, the value of $S_{n}$ depends on the environmental temperature. For a useful assessment of the properties of nuclei along the $\mathrm{r}$ process path, the value of the neutron separation energy should be known to within $k T \sim 100 \mathrm{keV}$, corresponding to the temperature of the r-process environment.

In other astrophysical applications, nuclear masses provide insight into the thermodynamics of the environment. Knowing the masses of nuclei involved in a reaction will allow for a calculation of the energy released in a reaction, which can provide information on environmental heating. Examples where this might be important include neutron star crust heating, ${ }^{2,3}$ x-ray burst thermal properties ${ }^{4}$ stellar heating, and explosive nucleosynthesis.

\subsection{Example: Nuclear Masses of Neutron-Rich Nuclei and the Astrophysical $r$ Process}

The astrophysical $r$ process is mentioned here as one example of an astrophysical site in which nuclear mass measurements may be useful. In the approximation of $(\mathrm{n}, \gamma) \rightleftharpoons(\gamma, \mathrm{n})$ equilibrium, corresponding to a the classical $\mathrm{r}$ process, nuclear masses can be used for a direct determination of neutron separation energies $S_{n}$. Though the canonical r-process may only have a limited contribution to galactic abundances, nuclear masses are still useful for understanding shell structure far from stability ${ }^{5}$ for any r-process scenario. Minor shell closures, the disappearance of shells, and the appearance of new shell structure can all inform theoretical development and evaluations of r-process sites.

Much of our current understanding of nuclei along the r-process path comes from extrapolating current mass models to the r-process progenitor nuclei. Of course, in the absence of actually measuring r-process progenitor nuclei, pushing studies of nuclear properties to more neutron-rich nuclei is advantageous. Thus, there are compelling reasons to study nuclei with increasing neutron richness as any extrapolation will then become more robust. These studies may be applicable to constraining mass models used in the r-process.

The impacts of nuclear mass uncertainties on an understanding of the $r$ process have been studied in detail in prior work. ${ }^{6-8}$ Shell structure far from stability is important to understand, but recent work has provided some interesting results which also indicate that the uncertainties in masses of the rare-earths must be constrained to better understand production of these elements. ${ }^{7,9}$

Mass models used to determine $S_{n}$ for neutron-rich nuclei can disagree significantly. Some mass models predict smooth trends in $\mathrm{S}_{n}$ along isotopic lines while 


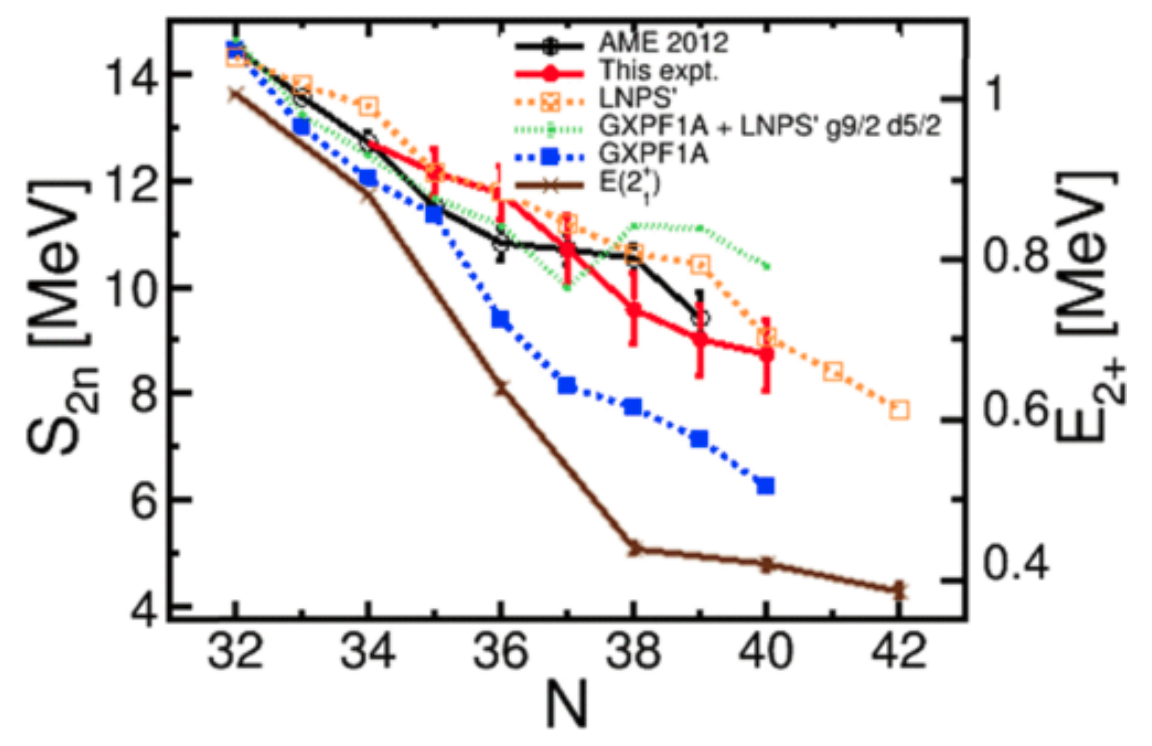

Fig. 1. Two-Neutron Separation energies for calcium nuclei (with the energy of the $2^{+}$yrast state indicated) for recent experimental work ${ }^{3}$ compared to various evaluations. Used with permission. ${ }^{3}$

others may predict shifts in the slope of $S_{n}$ as nuclei move further from stability, correlating with nuclear shape changes. Regions of "shape coexistence" may exist in the table of isotopes where nuclei along isotopic lines go from being prolate to oblate (or vice versa). Of course, different models result in different astrophysical results in various scenarios.

As an example of the importance of mass measurements, consider Figure 1, which shows $S_{2 n}$ trends along the Ca isotopic chain for experimental work compared to various theoretical models. The differences become more pronounced with neutron richness. This is not surprising as models can only be compared to and optimized with known masses. Mass measurements are necessary to constrain models for more exotic nuclei. Along isotopic chains, even measuring masses that are one nucleon beyond known masses can provide significant constraints on existing models.

Good overviews of nuclear data needs for astrophysical applications can also be found in Refs. ${ }^{10-12}$ and. ${ }^{13}$

\section{Techniques}

Techniques for measuring nuclear masses include the use of Penning traps, beamline TOF techniques, and storage rings. While numerous mass measurement techniques are available, ${ }^{14}$ we concentrate on those currently used for mass measurements of exotic nuclei. Each technique has its own advantages and disadvantages. Mass 


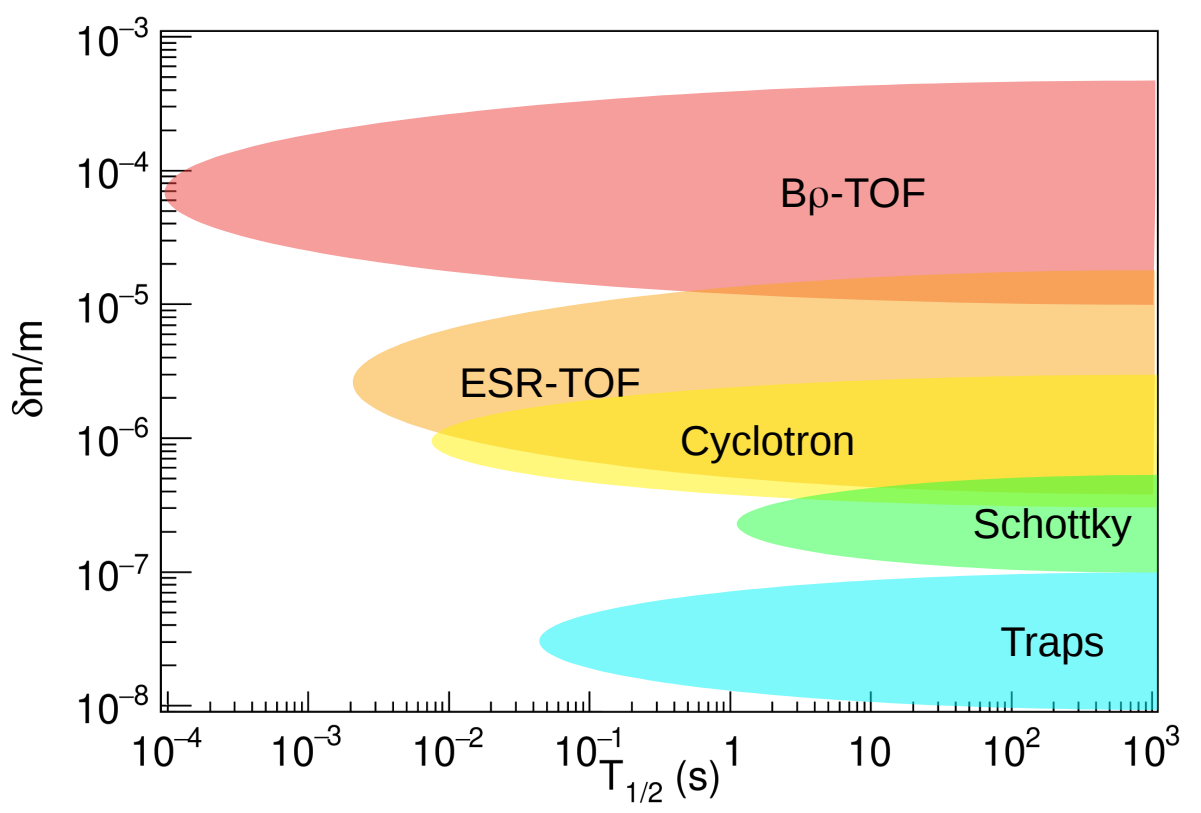

Fig. 2. Approximate resolutions and lifetimes of various mass measurement techniques. ${ }^{10}$ Used with permission. ${ }^{10}$

measurement techniques are limited by the half-lives of nuclei they are capable of measuring. Likewise, each technique has a mass resolution limit. The approximate range of lifetimes and mass resolutions accessible by various techniques is shown in Figure 2.

Mass measurements of exotic nuclei with radioactive ion beams (RIBs) come with constraints ubiquitous to the fact that unstable beams are being used. Using RIBs to measure masses means that any method used must be able to measure potentially short-lived nuclei, and these methods are also constrained by any rate limitations of the facility that produces these nuclei. Methods adopted to measure masses are specialized to deal with one or both of these constraints.

Direct measurements of nuclei include frequency-based and time-based measurement. ${ }^{11}$ Of the frequency-based measurements, Penning traps and storage rings are the most developed and well-known techniques. Time-of-flight (TOF) measurements vary in technique, but all rely on measuring the flight time of nuclei of unknown mass between two points in a beam path of known rigidity and comparing to that of nuclei for which the masses are known.

Given the number of techniques and efforts to measure nuclear masses with greater precision, constraints on nuclear masses and mass models for nuclei approaching the r-process path has seen significant progress in the past decade. A 

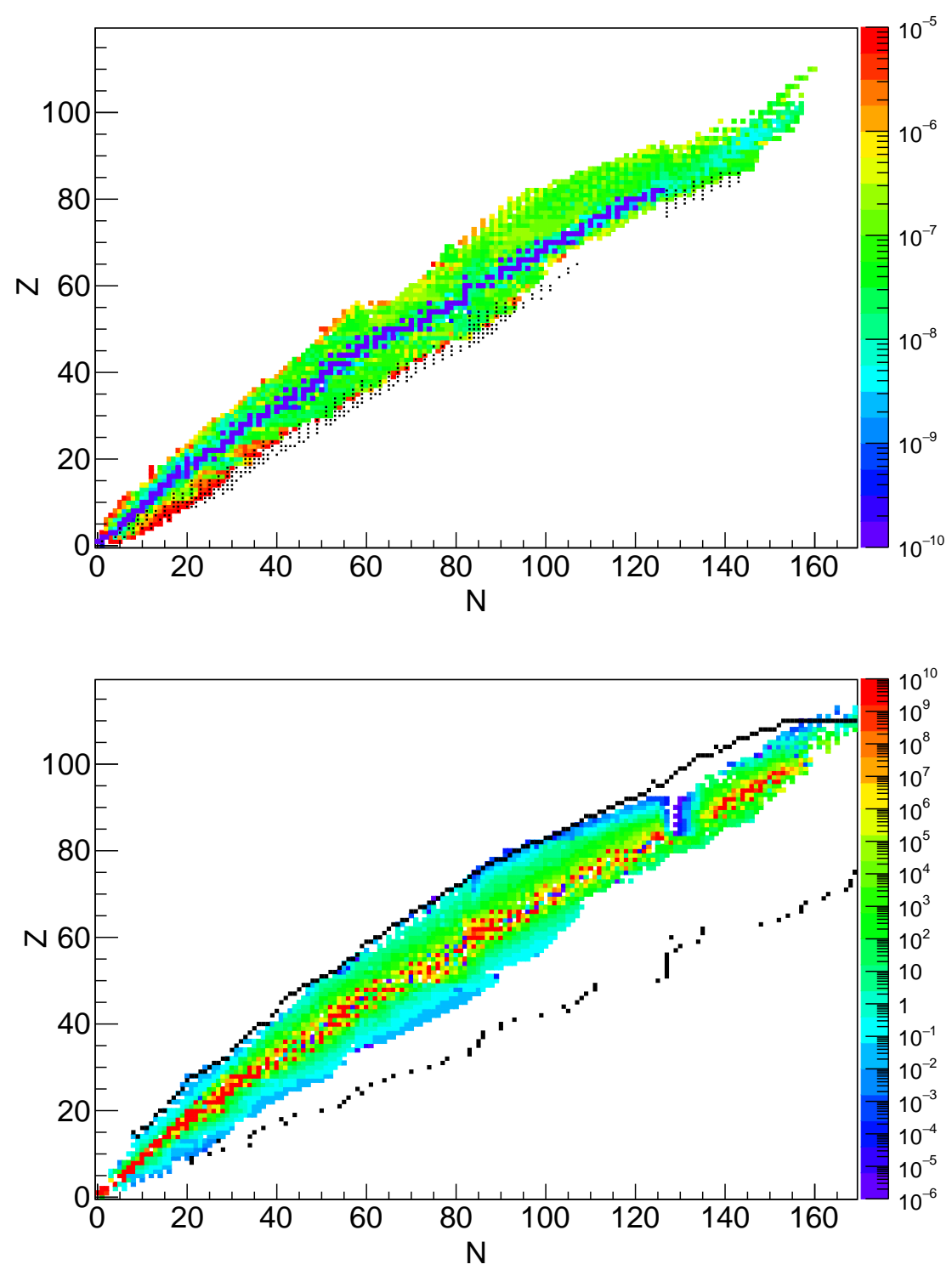

Fig. 3. Top: Relative uncertainties $\delta \mathrm{m} / \mathrm{m}$ of nuclear measured nuclear masses. ${ }^{15,16}$ Bottom: Measured decay rates of nuclei $\left(\mathrm{s}^{-1}\right)$. The approximate location of the r-process path is shown in the top figure, and the approximate locations of the drip lines are shown on the bottom. ${ }^{10}$ Used with permission. ${ }^{10}$

representation of the relative uncertainty in nuclear mass measurements as of the AME2016 $6^{15,16}$ update is shown in Figure 3. While the masses of most r-process nuclei have yet to be studied, progress continues to be made. 


\subsection{Penning Traps}

Perhaps one of the more precise methods for nuclear mass measurements is the Penning trap. This device functions by creating a longitudinal uniform magnetic field in a quadrupole electric field. A schematic a Penning trap is shown in Figure $4 .{ }^{17}$ Penning traps have been used to measure atomic and nuclear masses with resolutions of $\delta m / m \lesssim 10^{-8}$ for nuclei with half-lives as low as a few ms. ${ }^{18}$ They are also useful for mass measurements of low intensity beams. (For excellent reviews of Penning trap mass spectrometry, see Refs. ${ }^{18}$ and. ${ }^{19}$ )

In Figure 4, if the magnetic field defines the $\hat{z}$ axis in cylindrical coordinates, $\mathbf{B}=B_{\circ} \hat{z}$, then a quadrupole electric potential is defined by: ${ }^{18}$

$$
\phi(r, z)=\frac{\phi_{\circ}}{4 d^{2}}\left(2 z^{2}-r^{2}\right)
$$

where $d$ is a dimension characterizing the trap. The magnetic field in this configuration confines ions in a plane perpendicular to $\hat{z}$. The radial motion of the ions in the trap are defined by this field. The quadrupole electric potential confines ions axially. The cyclotron motion of ions in the trap is then related to the magnetic field, where the cyclotron angular frequency, $\omega_{c}$, is:

$$
\omega_{c}=\frac{q B}{m}
$$

In addition to the cyclotron oscillation, the ion can oscillate axially as a result of the electric field. Its motion can be shown to be simple harmonic with a frequency: ${ }^{19}$

$$
\omega_{z}^{2}=\frac{q \phi_{\circ}}{m d^{2}}
$$

The cyclotron frequency in the absence of the external electric field is shown above. However, with the external electric potential, the equations of motion are solved to result in three independent eigenfrequencies. One of these is the axial oscillation $\omega_{z}$. Another is a modification of the cyclotron motion $\omega_{+}$, and a third is an oscillation due to $\mathbf{E} \times \mathbf{B}$ drift motion. The angular frequencies $\omega_{ \pm}$are: ${ }^{19}$

$$
\omega_{ \pm}=\frac{\omega_{c}}{2} \pm \sqrt{\left(\frac{\omega_{c}}{2}\right)^{2}-\left(\frac{\omega_{z}}{\sqrt{2}}\right)^{2}}
$$

where $\omega_{+}$is known as the modified or reduced cyclotron frequency and $\omega_{-}$is known as the magnetron frequency. It's instructive to note that $\omega_{c}>\omega_{+} \gg \omega_{z}>\omega_{-}{ }^{18}$ and that $\omega_{c}=\omega_{+}+\omega_{-}$. The eigenmotions defined by $\omega_{z}$ and $\omega_{ \pm}$are shown in Figure 4.

An example of a typical mass measurement with a Penning trap is described in Refs. ${ }^{17}$ and. ${ }^{18}$ A typical measurement using a Penning trap would involve first cooling the ions to energies of a few $\mathrm{keV}$ using degraders. Gas cells are commonly used as degraders owing to their excellent uniformity and adjustability. The ions are then electrostatically guided to the trap, where their motion can be excited through resonance frequencies with $\mathrm{RF}$ fields. At the resonant cyclotron frequency, the ions 
are excited and then extracted from the trap, where their energy can be measured via time-of-flight from the trap to a TOF detector. The TOF measurement need not be as precise as the resonance frequency measurement, as one is only looking for the frequency at which the ion energy results in a resonance due to the cyclotron resonance being set up (and, hence, the TOF at resonance is dramatically different that off resonance). A resolution of a few ns is sufficient. ${ }^{17}$

The time it takes to complete this process of cooling, exciting, extracting, and measuring TOF is the major limitation of mass measurements using Penning traps. Such measurements typically consume a few 10s to 100s of ms, placing a lifetime limit on the isotopes that can be measured. This tends to limit the range of isotopic species that can be measured. However, the excellent precision is extraordinarily useful as Penning trap mass measurements can be used as calibration points for possibly lower-resolution techniques that can effectively measure masses of more exotic species.
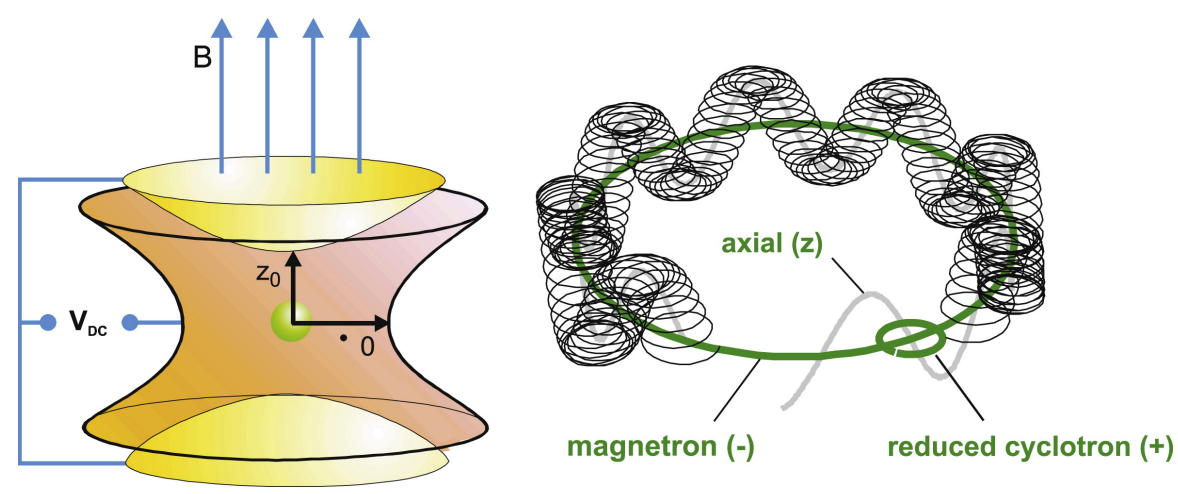

Fig. 4. Left: Schematic of a Penning trap spectrometer showing the electric field plates and the magnetic field. Right: Example of the path of an ion in a Penning trap ${ }^{17}$ showing the three motions associated with the eigenfrequencies described in the text. Used with permission. ${ }^{17}$

A number of trap facilities worldwide have measured both proton-rich and 
neutron-rich nuclei with excellent accuracy. Because of the lifetime limitations of traps, extremely exotic species - such as those on the r-process path - have fewer measurements. However, where measurements exist, the resolution is sufficient to use these as calibration points for less-precise methods that can measure the masses of the more exotic species.

Figure 5 shows the masses of various nuclei that have been measured with Penning traps. A brief summary of a representative sample of Penning trap devices is given below.

\subsection{1. $C P T$}

The Argonne National Laboratory Canadian Penning Trap (ANL-CPT) has been inserted as a functional component of the ATLAS linac. ${ }^{20-22}$ Significant progress has been made with the CPT to measure the masses of isotopes along the $\mathrm{N}=\mathrm{Z}$ line. However, measurements have also been made of the masses of nuclei approaching the r-process path. ${ }^{22}$ This device is capable of mass accuracies of $\delta m / m \sim 10^{-8}$.

In addition to the Penning trap, the CPT is coupled to a radio-frequency quadrupole (RFQ) trap. Species are produced in the ATLAS facility and analyzed in the ANL Enge split-pole spectrograph. Prior to trap injection, nuclei are cooled in a gas cooler/buncher system.

The recent CARIBU upgrade at ANL has increased the reach of nuclei available at the CPT. ${ }^{22}$ Recently, 40 neutron-rich nuclei with $51 \leq Z \leq 64$ have been measured, along with Te and Sb nuclei along the r-process path. ${ }^{22}$

\subsubsection{ISOLTRAP}

Over 400 nuclear masses have been measured with the ISOLTRAP tandem Penning trap facility located at the online isotope separator ISOLDE at CERN. Nuclei with lifetimes as low as $\sim 50 \mathrm{~ms}$ have been measured with typical accuracies of $\delta m / m \sim 5 \times 10^{-8} .23,24$

The ISOLTRAP facility is enhanced with the addition of a multi-reflection timeof-flight (MR-TOF) mass separator and spectrometer. ${ }^{24}$ (See $\S 2.5$ for a brief description of the MR-TOF method.) The primary purpose of this is to provide an additional level of beam purification. Because of the exceptional beam purity provided, the ISOLTRAP facility is also used to measure half-lives of exotic nuclei in a background-free environment. ${ }^{25}$ The half-life of ${ }^{27} \mathrm{Na}$ was recently measured with this setup. ${ }^{25}$

While the list of nuclei measured with ISOLTRAP is extensive, notable cases include the masses of nuclei between between ${ }^{17} \mathrm{Ne}$ and ${ }^{232} \mathrm{Ra} .{ }^{26,27} \mathrm{In}$ addition, ISOLTRAP has been used to measure the masses of nuclei near the $N=82$ shell closure. Current results are also pushing the limits of precise neutron-rich masses past the $Z=82$ shell closure. This is challenging given the lifetime limitations off Penning traps and the lifetimes of r-process progenitor nuclei. 


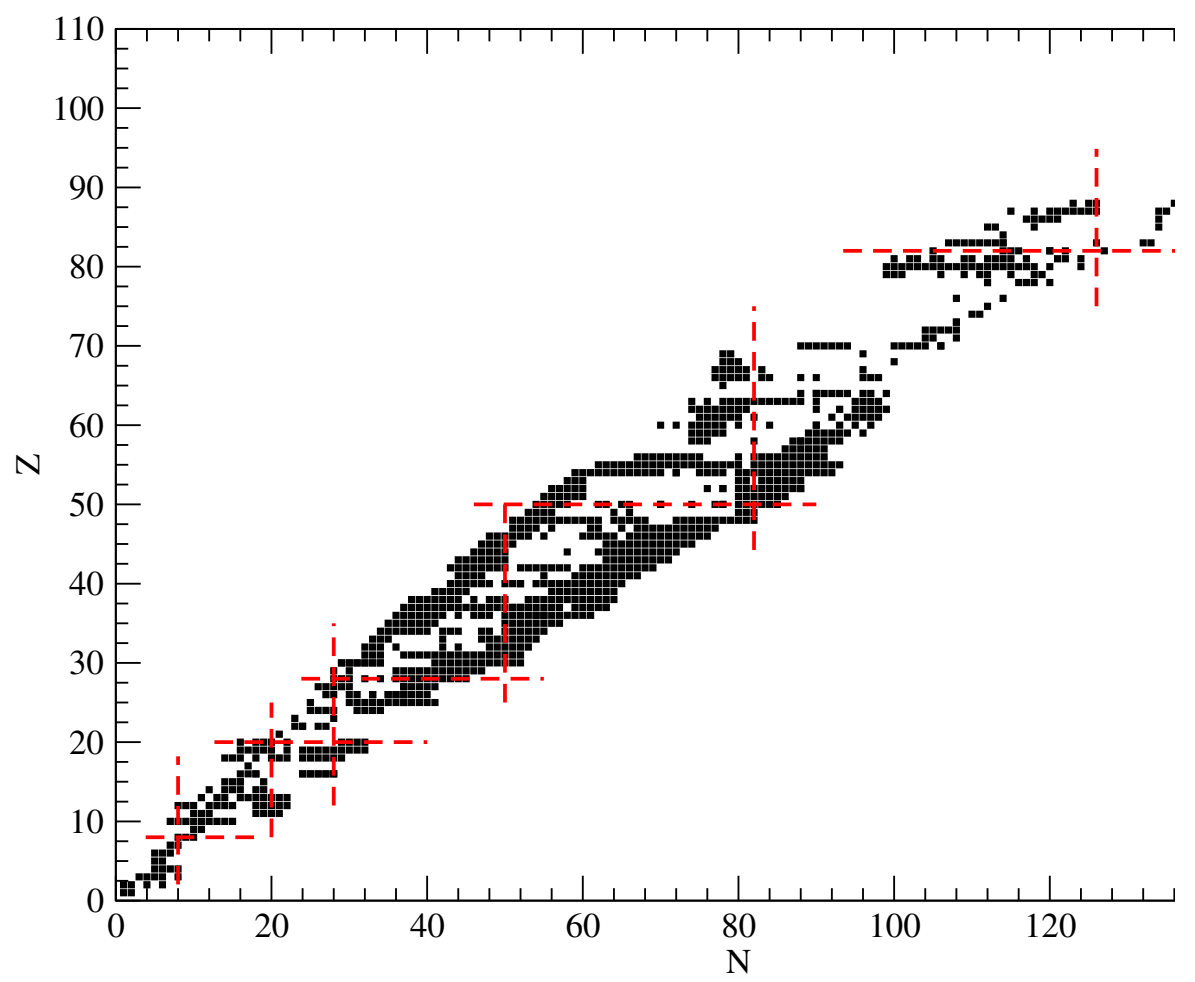

Fig. 5. Isotopic species that have been measured with Penning traps. ${ }^{18}$ Isomers and charge states are not distinguished in this figure. Nuclear shell closures are indicated by the dashed lines. 


\subsubsection{JYFLTRAP}

JYFLTRAP is located at the IGISOL facility at the University of Jyväskylä. ${ }^{28-31}$ It has achieved mass accuracies near $50 \mathrm{keV}$ and generally produces results with $\delta m / m \sim 10^{-8} \cdot{ }^{32,33}$ To date, it has measured nearly 400 masses up to $\mathrm{A} \approx 120$. Of these, roughly 300 are masses of neutron-rich nuclei. ${ }^{28,34-42}$ These include masses near the $\mathrm{Z}=50 \mathrm{r}$-process waiting point as well as many of the neutron-rich rare earth nuclei.

\subsection{4. $L E B I T$}

The Low Energy Beam and Ion Trap (LEBIT) facility has been a leader in trap facilities in the USA. The LEBIT trap takes advantage of the radioactive ion beam production capabilities of the National Superconducting Cyclotron Laboratory by slowing the fragmented fast beams prior to trap injection. ${ }^{17,43}$ Fragments produced in the NSCL coupled-cyclotron facility (CCF) and separated in the A1900 fragment separator are slowed in the NSCL gas stopping system. ${ }^{17}$ The process of combining the analysis beam, gas stopping system, and trap is essential for utilizing a trap in a facility that provides a large variety of fragmentation beams.

Slowing and stopping of the beams in LEBIT is accomplished with a linear gas stopper and cyclotron gas stopper. ${ }^{44}$ Prior to injection into the Penning trap, a beam accumulator and buncher cools the beam. ${ }^{45}$

Many of the masses which are difficult or impossible to produce at ISOL facilities can be produced at the NSCL, making LEBIT complementary to existing facilities. As a result, the LEBIT facility has measured the masses of over 50 isotopes with high precision, with a concentration on the proton-rich nuclei. As the NSCL transitions to the Facility for Rare Isotope Beams (FRIB), new opportunities may be available for LEBIT. ${ }^{46}$

\subsubsection{SHIPTRAP}

The GSI SHIPTRAP facility has concentrated primarily on proton-rich nuclei. It has also excelled at measuring the masses of more massive nuclei. ${ }^{26}$ Between ISOLTRAP, SHIPTRAP, and JYFLTRAP, devices on the European continent have measured a very large range of masses with the Penning trap technique. It consists of a two-stage trap system with the intent of searching for super-heavy elements. It is thus optimized for the transmission and measurements of transuranic nuclei with $Z \geq 92$, filling a niche among the trap facilities. ${ }^{47}$

Early measurements with SHIPTRAP include ${ }^{147} \mathrm{Ho}^{+},{ }^{147} \mathrm{Er}^{+}$, and ${ }^{148} \mathrm{Er}^{+48}$ with latter measurements prepared to examine the endpoint of the rp-process. ${ }^{49}$

\subsubsection{TITAN}

The TITAN facility (TRIUMF's Ion Trap for Atomic and Nuclear science) consists of several Penning traps. Recently, TITAN underwent an upgrade to install a charge 
breeder in its electron beam ion trap. ${ }^{50}$ This allows for the production of higher charge states, resulting in improved mass resolution.

For decay spectroscopy, the facility utilizes photon counters and seven $\mathrm{Si}(\mathrm{Li})$ detectors for simultaneous in-trap decay spectroscopy experiments. ${ }^{50-54}$ TITAN has been used for nuclear mass measurements and mass model constraints, ${ }^{55}$ though its primary purpose is determinations of the $V_{u d}$ CKM matrix elements. Precise measurements of the masses of parent and daughter nuclei are necessary for precise determinations of $V_{u d}$.

Recently, TITAN has been used to measure the neutron-rich nuclei ${ }^{98,99} \mathrm{Rb}$ and ${ }^{98-100} \mathrm{Sr}^{56}$ This is useful for nuclei approaching the r-process path. In addition, the masses of nuclei approaching the $\mathrm{N}=82$ closed shell, ${ }^{125-127} \mathrm{Cd}$ have also been measured. ${ }^{57} \mathrm{~A}$ notable feature of TITAN is its ability to measure nuclear masses with very low half-lives, such as the mass of ${ }^{11} \mathrm{Li}$, with $t_{1 / 2}=8.8 \mathrm{~ms}^{58}$

\subsection{Other Trap Facilities}

While the Penning trap itself is small and relatively straightforward to manufacture, the equipment necessary to successfully operate the trap, including the field generators, beam coolers, power supplies, and RF sources makes the operations of the trap precise and the requirements exacting.

However, multiple traps have been installed about the world in addition to those described above. Other successful Penning trap programs and those planned or under construction include the Florida State University trap ${ }^{59}$ SMILETRAP ${ }^{60}$ MATS ${ }^{61}$ PIPERADE ${ }^{62}$ WITCHTRAP ${ }^{63}$ REXTRAP, ${ }^{64}$ TRIGA-TRAP,${ }^{65}$ the Mainz trap,${ }^{66}$ HITRAP,${ }^{67}$ PENTATRAP, ${ }^{68}$ MLLTRAP, ${ }^{69}$ the Lanzhou trap,${ }^{70}$ and the RIKEN trap. ${ }^{71}$ These are dedicated to various studies involving the measurements of nuclear masses as well as fundamental properties of matter and antimatter.

\subsection{Time-of-Flight Methods}

As the name implies, time-of-flight (TOF) methods are used to determine nuclear masses by measuring the flight time of nuclei between two points in an spectrometer. The spectrometer is calibrated with the masses of known nuclei. Thus, the accuracy of mass measurements with TOF methods depends on the uncertainties of the calibration masses, though these uncertainties are not necessarily the largest contributor to the total measurement uncertainty.

Mass measurements determined via TOF are generally less accurate than those determined with traps. However, while traps are limited by the lifetime of the measured nuclei, the lifetime of nuclei measured with TOF method is only limited by the time for nuclei to traverse the flight path of the spectrometer used. This is generally well under $1 \mu \mathrm{s}$.

Beamlines used in the TOF method can consist of a "tagging section" in which flight time is measured between two distinct points in a spectrometer. Alternatively, 
closed-loop flight paths (rings) allow for the particle of interest to pass the same point in the beamline multiple times. The ring method is generally more accurate as measurements can be averaged over multiple particle passes, and systematic error can be reduced as only a single TOF detector is necessary.

\subsubsection{Rigity-Based TOF Measurements}

The $\mathrm{B} \rho$-TOF method is used to measure nuclear masses through the measurement of the flight-time of nuclei between two points in a beam facility; ${ }^{12}$ thus, it utilizes a tagging section of a spectrometer. The tagging section is typically $\sim 100 \mathrm{~m}$ in length in facilities employing this method. This experimental setup is conceptually straightforward and the associated device parameters can be varied significantly to suit the goals of a experiment. However, though this method employs detectors typically used in a variety of nuclear physics experiments, they must be operated and understood very precisely.

In many measurements employing the $\mathrm{B} \rho$-TOF method, beamline diagnostics are used (or substituted with devices of higher precision). In a typical setup, two or more detectors are placed at the ends of the beamline tagging section to determine the flight time of short-lived species. The TOF measurement is complemented with a measurement of the particle rigidity $B \rho$, thus the reason for referring to this method as the $B \rho$-TOF method.

Radioactive ion beam accelerators are, to first order, momentum selectors. In the simplest approximation particle momentum is related to the magnetic field and turn radius in the bend of a beam path through the field. The relationship between particle mass and rigidity can be easily derived:

$$
\frac{B \rho}{\gamma}=\frac{m v}{q}
$$

where $\gamma$ is the usual Lorentz factor for particles in the beamline. With an overall TOF uncertainty of $\sim 50 \mathrm{ps}$ (after correcting for path length via a measurement of the particle rigidity), and a TOF $\sim 0.5 \mu \mathrm{s}$, the uncertainty in the mass from the TOF is $\sim 10^{-4}$. Greater statistics can reduce the average statistical uncertainty to values typically $\sim 10^{-6}$ to $\sim 10^{-5}$. This corresponds to a mass resolution of $\sim 100$ $\mathrm{keV} / \mathrm{c}^{2}$, which is roughly the temperature, $k T$, of the r-process environment. This resolution is generally sufficient to constrain mass models far from stability.

The lifetime limitation of measured nuclei is due to the TOF between two detectors. Calibration of flight times with this method is accomplished by incorporating known "reference masses" in the mixed beam. Because the $\mathrm{B} \rho$-TOF method can measure shorter lifetimes, it can extend the range of known masses to more neutronrich nuclei. This method is also useful for measuring multiple masses simultaneously in a single experiment.

Reference masses for calibration of the method can be taken from masses measured with Penning traps. The major limitation in the limit of nuclei that can be measured is then the production rates of exotic beams. 
Particle rigidity with this method can be determined by measuring beam position at dispersive planes in the beamline. Additional complications of this method may be the incorporation of charge states (species that are not fully ionized or species in multiple ionization states) in the beam, which must be separated by measuring the energy deposition in a particle telescope at the end of the beamline. This method is sometimes referred to as the $B \rho$-TOF- $\Delta E$ method.

A representative sample of mass measurements at facilities incorporating the $B \rho$-TOF or $B \rho$-TOF- $\Delta E$ method are described below.

\subsection{2. $N S C L$}

Located in East Lansing, MI, the National Superconducting Cyclotron Laboratory (NSCL) in East Lansing, MI currently utilizes the coupled-cyclotron facility (CCF) and the A1900 spectrometer coupled to the S800 spectrometer to create a particle flight path of about $88 \mathrm{~m}$. A schematic of a typical NSCL TOF mass measurement experiment is shown in Figure 6.

The TOF detectors are placed at the A1900 extended focal plane and the focal plane of the S800. Particle rigidity is measured at the target position of the S800. ${ }^{72,73}$ (See Fig. 6.) Figure 6. For such a setup, the tagging section begins at the end of the A1900 spectrograph ${ }^{74}$ and ends at the end of the S800 spectrograph. ${ }^{75}$ Both spectrographs are matched in rigidity to allow the particles of interest to pass with a minimum loss of beam intensity. A point of maximum momentum dispersion is located in the S800, usually at the target location. While the S800 can be operated with a dispersive plane at the end station, running in the mode with the dispersive plane at the target location and a focus at the S800 terminus allow for the use of small TOF timing scintillators in the device, which can reduce the uncertainty and subsequent necessary corrections to particle position in the scintillators.

In order to measure the particle position at the $\mathrm{S} 800$ focus, the detector used must have as little material as possible interfering with the beam to reduce the amount of energy straggling in the beam, which adds an additional uncertainty. Ideally, any detector used should be placed as close to the end of the tagging section as possible. The reason for this is because the uncertainty induced in the velocity, $\delta v$ will result in an uncertainty in the $\delta$ TOF of the beam particles. If the length between the rigidity measurement and the end of the tagging section, $d$, is shorter, the shift in TOF is then $\delta \mathrm{TOF}=d / \delta v$. A detector of choice for such a task would be a micro-channel plate (MCP) based on secondary electron emission. ${ }^{76,77}$ Such devices can introduce $\sim 50 \mu \mathrm{g} / \mathrm{cm}^{2}$ of material into the beamline while providing very fast $(\lesssim 1 \mathrm{~ns})$ time resolution and excellent position resolution $\left(\sigma_{x} \sim 500 \mu \mathrm{m}\right)$.

By the early half of the decade starting in 2020, the NSCL will transition to the Facility for Rare Isotope Beams (FRIB). As a result the production rates of more exotic nuclei will make it possible to feasibly measure nuclear masses farther from stability using the $\mathrm{B} \rho$-TOF technique. Predicted production rates for nuclei at the 


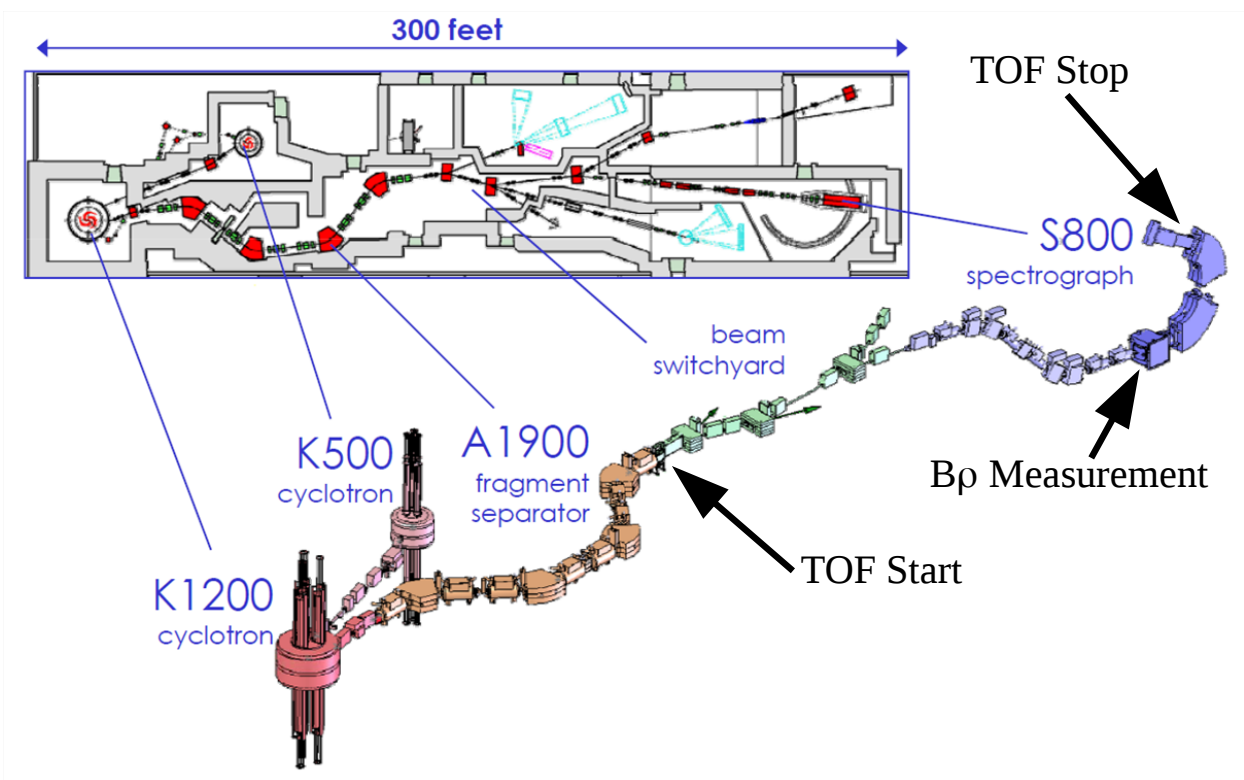

Fig. 6. Schematic of the TOF-B $\rho$ experimental setup at the NSCL facility. ${ }^{78}$

FRIB are shown in Figure $7 .{ }^{79}$ It is predicted that nuclear masses for nuclei well past the second r-process peak into the rare earth region will be possible. ${ }^{80}$ In addition, the rigidity resolutions afforded by the planned High Resolution Spectrometer (HRS), should allow for sensitive rigidity corrections for TOF measurements at the HRS. ${ }^{81}$

Over the past several years, the NSCL has developed several mass measurement experimental programs. ${ }^{82}$ In particular, neutron-rich nuclei of increasing mass have been a target of many recent experiments. ${ }^{3}$ Recent published results include the measurements of ${ }^{59-64} \mathrm{Cr}$ and neutron-rich Ar, Sc, Mn, and Fe relevant to crustal heating in neutron stars. ${ }^{2,3}$ At the time of this writing, analysis continues on mass measurements to evaluate the shape coexistence of nuclei near the r-process path during the early onset of the r-process. Additional measurements using this technique at the NSCL have determined the masses of ${ }^{61} \mathrm{~V},{ }^{63} \mathrm{Cr},{ }^{66} \mathrm{Mn}$, and ${ }^{74} \mathrm{Ni}^{83,84}$ Resolutions achieved were $10^{-4}$.

\subsubsection{Spectromètre à Perte d'Energie du Ganil (SPEG)}

Located at GANIL in Caen, France, the high-resolution SPEG contains an $82 \mathrm{~m}$ flight path coupled to an $\alpha$ spectrometer (so named because the shape of the spectrometer resembles the Greek " $\alpha$ "). The mass measurement program associated with the SPEG device has seen a successful program covering decades. ${ }^{85-87}$

While the NSCL program uses plastic scintillators as the timing detectors of the 


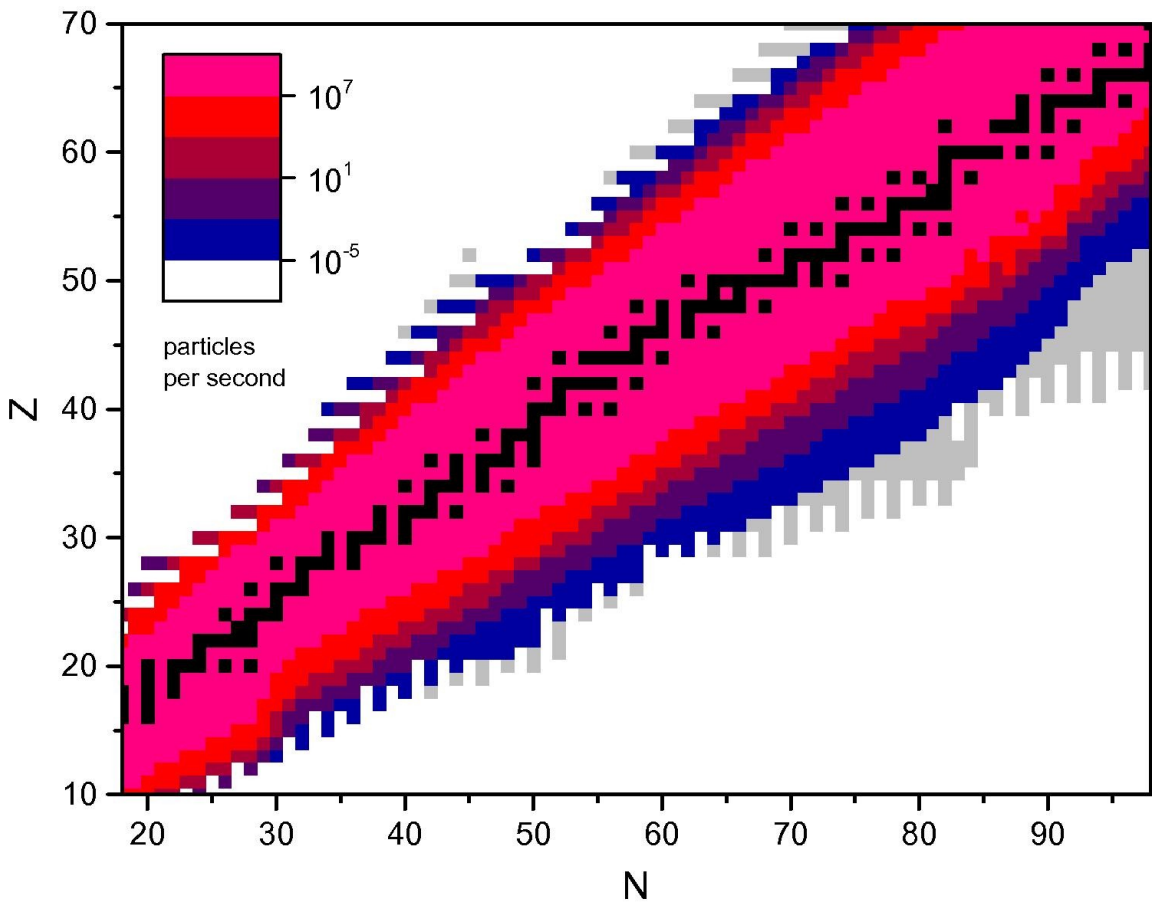

Fig. 7. Predicted isotopic production rates at the Facility for Rare Isotope Beams. ${ }^{79}$ Used with permission. ${ }^{79}$

tagging section, the SPEG program uses microchannel plate detectors ${ }^{4,76}$ which have an intrinsic time resolution of $\sim 100 \mathrm{ps}$ (FWHM). Two independent rigidity measurements are also employed; one is at the dispersive focal plane while the other is made after the TOF tagging section.

Many of the SPEG measurements have concentrated heavily on neutron-rich nuclei with $\mathrm{N}=16,20$, and 28 . This may be of particular importance for the "light element primary process" (LEPP).

SPEG measurements of neutron-rich masses near the $\mathrm{N}=20$ shell closure have been used to verify a region of shape coexistence about $\mathrm{N}=20$ and $\mathrm{N}=28$ nuclei. These measurements targeted the masses of nuclei with $29 \leq A \leq 47 .{ }^{88}$ The SPEG programs has also resulted in mass measurements of several light, neutron-rich nuclei ${ }^{89,90}$ with resolutions as low as $\sim 10^{-5}$. These include measurements of ${ }^{29,30} \mathrm{Ne}$, ${ }^{34,35} \mathrm{Mg},{ }^{36,37} \mathrm{Al}$, and ${ }^{31-33} \mathrm{Na} .{ }^{91,92}$ Additional successful attempts have been made to measure masses of nuclei closer to the neutron drip line, ${ }^{93,94}$ and significant improvements of neutron-rich masses in the mass region $\mathrm{A} \sim 10-50$ have been made including measurements of ${ }^{19} \mathrm{~B},{ }^{22} \mathrm{C},{ }^{29} \mathrm{~F},{ }^{31} \mathrm{Ne},{ }^{34} \mathrm{Na}$, and other light nuclei. ${ }^{95}$ These measurements were instrumental in confirming the halo structure of several 
of the light nuclei in this region. The $\mathrm{C}$ and $\mathrm{B}$ nuclei are presumed to be at the neutron drip line.

\subsubsection{Other Facilities}

The SHARAQ spectrometer at the Radioactive Ion Beam Factory in Saitama, Japan has recently been completed. ${ }^{96-98}$ This creates a $105 \mathrm{~m}$ TOF tagging sections. Radiation-hard diamond detectors are used to sustain high rates with excellent time resolution. ${ }^{99}$ The SHARAQ spectrometer is very high resolution, allowing for precise rigidity measurements. Recently, the masses of ${ }^{55,56} \mathrm{Ca}$ have been measured with a resolutions of $\sigma \sim 150 \mathrm{keV}$ and $234 \mathrm{keV}$ respectively.

Another example of a promising facility is the collaborative effort between Beihang University and the Heavy Ion Reseach Facility in Lanzhou (HIRFL). ${ }^{100}$ Detector development by this collaboration has resulted in fast plastic scintillators for timing purpose. These have an intrinsic time resolution of $5.1 \mathrm{ps} .{ }^{101}$ The HIRFL collaboration has also worked to develop high resolution multi-wire proportional counters (MWPCs) with intrinsic position resolutions of $1 \mathrm{~mm}$ for rigidity measurements.

For light-Z neutron-rich nuclei, the Time-of-Flight Isochronous (TOFI) spectrometer ${ }^{102-104}$ is designed for isochronous mode operation. In isochronous mode, the spectometer is set such that all species have the same flight time while the momentum is used exclusively to determine mass. Thus, slower-moving ions travel a shorter path, requiring precise position measurements at the dispersive focal planes. This measurement is also used by rings (described in $\S 2.4$ ). The TOFI spectrometer has been used to measure the masses of neutron-rich isotopes from $\mathrm{Li}$ to $\mathrm{P}^{102,104}$ with resolutions of 200 to $900 \mathrm{keV}$.

\subsection{Storage Rings Methods}

Much like the $\mathrm{B} \rho$-TOF method, storage rings operate based on the time of flight of the nuclei being measured. However, because the flight path is a closed path, storage rings are capable of measuring particle passage at one point in the ring as the particles pass that point ${ }^{105,106}$ (though measurements at more than one point can be made as well). This method has several advantages. One advantage is that only a single timing detector is necessary in the particle flight path. Of course, the energy loss of the particle in the detector must be kept to a minimum, but systematic errors induced by multiple particles can be reduced. A second very powerful advantage is that the storage ring is able to measure the a particle several times as it traverses the loop multiple times prior to decay. An example of a storage ring is illustrated in Figure $8^{107}$ showing the GSI Experimental Storage Ring (ESR). Here, multiple components of the ring are indicated, including the injection mechanism and measurement locations.

However, unlike the $\mathrm{B} \rho$-TOF method, because they make multiple measurements of the same particle, storage rings are typically classified as frequency-based 


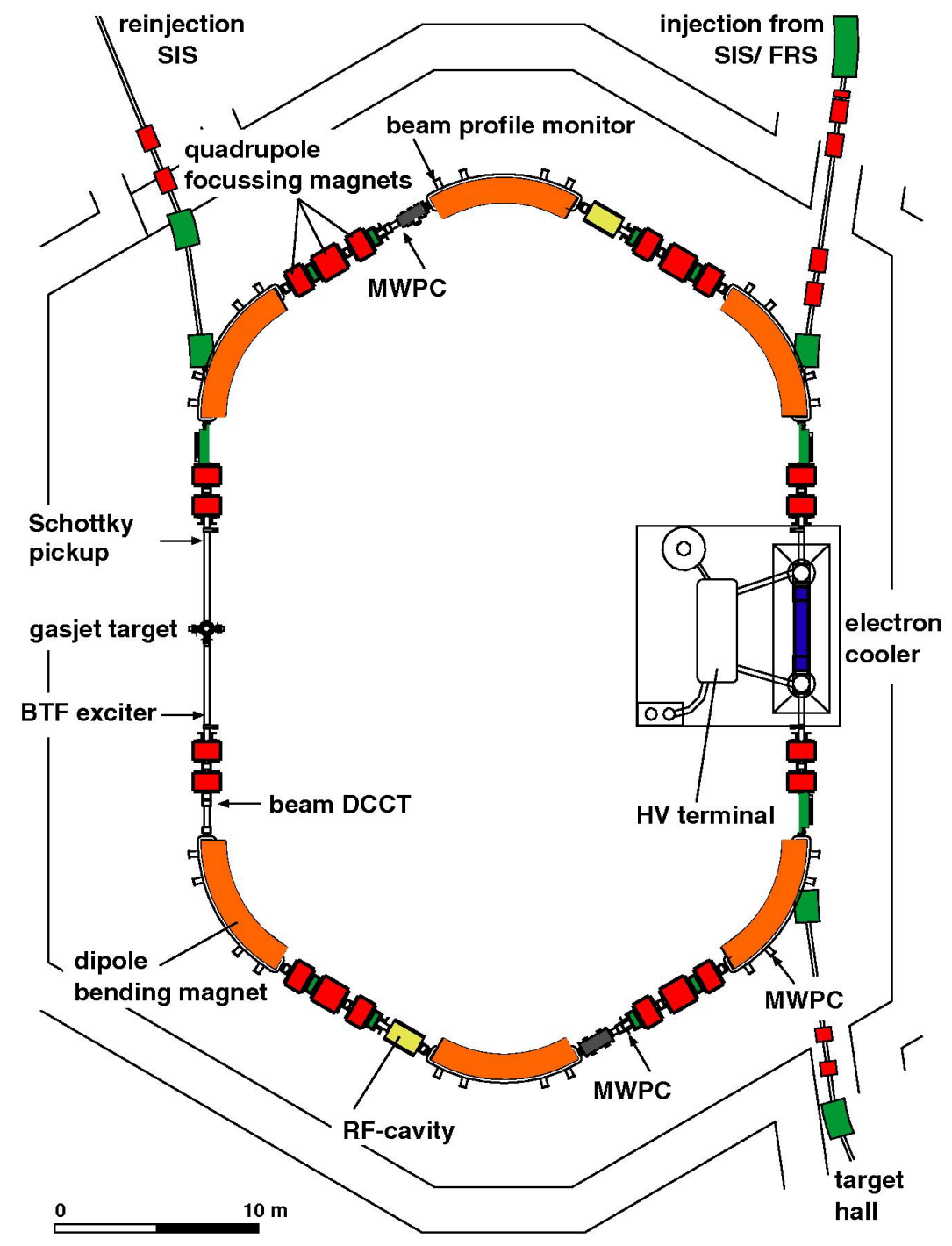

Fig. 8. An example layout of the GSI ESR storage ring. ${ }^{107}$ Used with permission. ${ }^{107}$

measurements in that they make multiple measurements of a particle's passage about the ring in a time period. It has been shown that the relationship between the number of circuits that a particle traverses about the ring is related to its 
mass by the measurement of revolution frequency of ions in a ring of circumference C: ${ }^{107,108}$

$$
f=\frac{\beta c}{C}
$$

For ions in the storage ring, the deviation in individual frequency measurements, $\delta f$, is:

$$
\begin{aligned}
\frac{\delta f}{f} & =\frac{\delta \beta}{\beta}-\frac{\delta C}{C} \\
& =\frac{1}{\gamma^{2}} \frac{\delta \beta \gamma}{\beta \gamma}-\alpha_{p} \frac{\delta p}{p} \\
& =\left(\frac{1}{\gamma^{2}}-\alpha_{p}\right) \gamma^{2} \frac{\delta \beta}{\beta}-\alpha_{p} \frac{\delta(m / q)}{(m / q)}
\end{aligned}
$$

with $\gamma$ the Lorentz factor. The term $\alpha_{p}$ is known as the "compaction factor" which is the ratio between the relative orbital path length and the relative momentum $\delta L / L=\alpha_{p} \delta p / p$ and results from the magnetic parameters in the storage ring. The third line above results from inserting $p=m \beta \gamma c$ into the second line and expanding.

From Equation 7, it can be seen that the frequency width of a single species is related to the mass uncertainties in a single species. Thus, more accurate frequency measurements result in more accurate mass measurements.

The resolution of a storage ring can be improved in two ways. The first is by tuning the beam parameters such that $\alpha_{p}=\gamma^{-2}$, so that the first term vanishes, known as "isochronous mode" in which the revolution frequency is constant for a nuclear species. ${ }^{109,110}$ This method works well for short-lived species, but has the drawback that it requires beam dispersions which can be large at even moderately relativistic settings.

Another way is to reduce the spread in beam velocity $\delta \beta / \beta$ through "cooling," effectively reducing $\delta \beta$ in Equation 7 . Here, the phase space of the beam is greatly constrained through a number of methods. ${ }^{111}$ The frequency of particle revolutions for multiple particles in the ring can then be analyzed as a Schottky spectrum, in which all species have the same average velocity, and shifts in frequency correlate with shifts in mass. ${ }^{112}$ Cooling is particularly necessary for longer-lived beams when the momentum dispersion increases due to collisions with other beam particles, beamline gas, space-charge effects, and beam target collisions. One example is that employed by the GSI-ESR and others in which a beam of co-moving electrons is injected into the primary particle beam. ${ }^{113-116}$ Coulomb interactions between the electrons and the beam result in a thermal equilibrium prior to the removal of the electrons from the beam, having absorbed some of the transverse momentum of the beam.

Because rings effectively count the revolutions of ions, they are also able to provide relatively accurate measurements of lifetimes by observing the revolution number at which the ion no longer exists. ${ }^{117}$ The lifetime of a single particle is then the number of revolutions a particle makes in the ring multiplied by the time per 
revolution. The measured lifetime then has a resolution that equal to the time for one revolution, $1 / f$. With modern rings, this is $\sim 2 \mu \mathrm{s}$.

A representative sample of storage ring facilities used for mass measurements is described in what follows.

\subsubsection{GSI-ESR}

The experimental storage ring located at GSI (GSI-ESR), which was described briefly previously and in Figure $8^{107,118}$ began operation in 1990. Particles analyzed in the ring are produced in the fragment separator (FRS) prior to injection into the ESR.

With a circumference of $108 \mathrm{~m}$ and a maximum magnetic rigidity of $10 \mathrm{Tm}$, the ESR can transmit $\mathrm{U}$ nuclei with a maximum energy of $560 \mathrm{MeV} / \mathrm{u}$ at a rotation frequency exceeding $2 \times 10^{6} \mathrm{~s}^{-1} \cdot{ }^{10,118}$

The ESR is capable of running in both Schottky mass spectrometry (SMS) (all particles ideally have the same velocity) or isochronous mass spectrometry (IMS) mode (all particles have the same frequency), both of which are described above. In SMS mode, particles in the beam are cooled to the central velocity of the beam, such that $\delta v / v \rightarrow 0$.

The ESR continues to push nuclear mass measurements towards the neutron drip line. For example, a recent measurement of the ${ }^{129-131} \mathrm{Cd}$ nuclei near the $\mathrm{N}=82$ waiting point was made with a mass accuracy $\delta m / m=2 \times 10^{-4} \cdot{ }^{119}$ From the perspective of the astrophysical $r$ process, this is useful for determining the strength of the $\mathrm{N}=82$ shell gap. Also, operating in IMS mode, mass measurements of the neutron-rich nuclei between Se and Ce were made using a fragmented ${ }^{238} \mathrm{U}$ primary beam. ${ }^{120}$ This measurement was important in extending the landscape of known nuclear masses by one additional neutron past stability. Recent measurements were also made for neutron-rich nuclei near Lu and Os in SMS mode. ${ }^{121}$ Other measurements include 35 mass measurements of neutron-rich nuclei between As and Xe. ${ }^{122}$ While many of the measured masses are not yet on the r-process path, they are useful in constraining mass models near the rare earth region. This is important as many mass models have significant disagreement in this region.

\subsubsection{HIRFL-CSRe}

A newer facility located at the Heavy Ion Research Facility in Lanzhou (HIRFL) ${ }^{123-128}$ the cooler-storage-ring (HIRFL-CSRe) has begun operation in the last decade with mass measurements of over 60 nuclei and 20 first time measurements. ${ }^{129-136}$ Both proton-rich and neutron-rich nuclei were re-measured or measured for the first time. Of these, a significant number of proton-rich $p f$-shell nuclei were measured for the first time at the CSRe with mass uncertainties $\delta m / m \sim 10^{-7}$ $-10^{-6}$. These measurements were made by running the CSRe in IMS mode. These are of particular interest for evaluating the effects of nuclear structure and electron- 


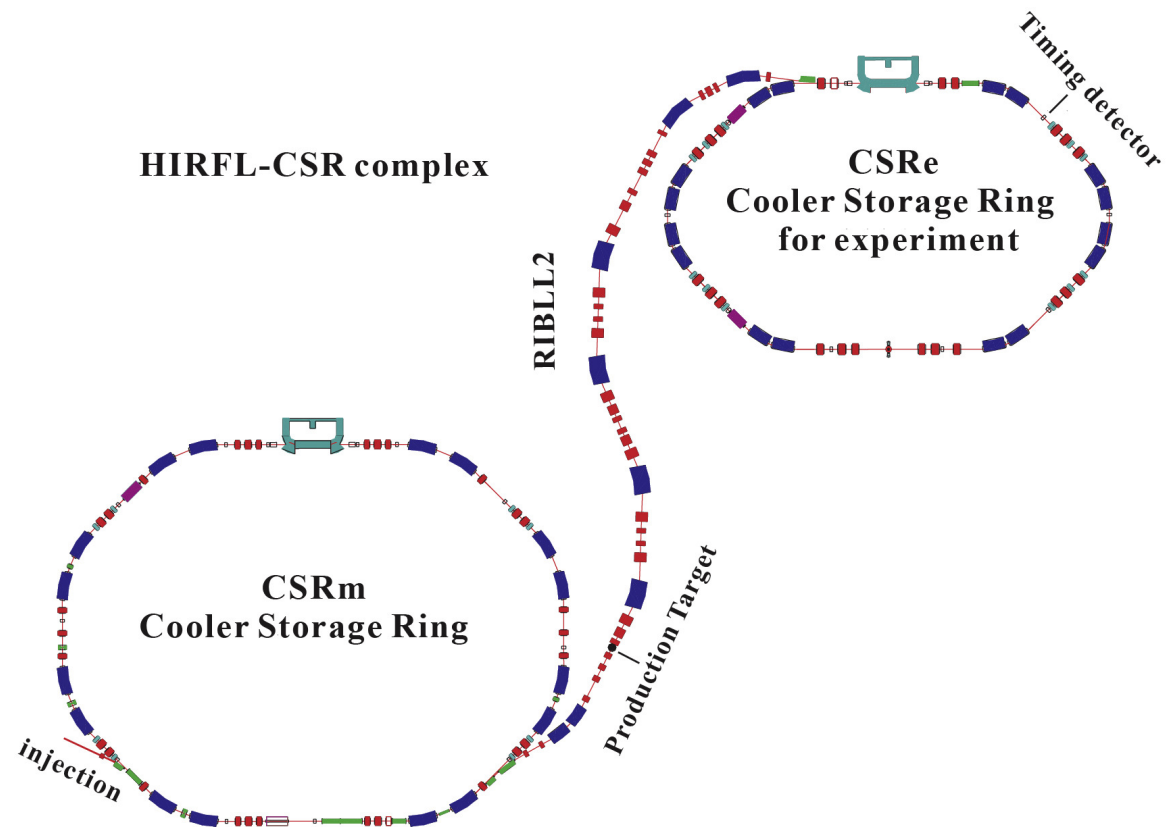

Fig. 9. Layout of the HIRFL-CSRe system, including the spectrometer to select ions, the production target, and the storage ring. ${ }^{133}$ The electron cooler is shown at the top of of the CSRe in the figure. Dual timing detectors can be used in the straight section of the ring opposite the cooler. Used with permission. ${ }^{133}$

capture properties of nuclei relevant to type Ia supernovae. In addition, as new masses are measured on or near the proton drip line, the structure of nuclei relevant to the astrophysical rp process can be better understood.

The CSRe is similar in structure to the GSI-ESR. An example of the complete beamline, including the injection system, is shown in Figure $9 .{ }^{133}$ Secondary beams are produced from a primary beam with energies of $350-480 \mathrm{MeV} / \mathrm{u}$ incident on a $10-15 \mathrm{~mm}$ Be production target. ${ }^{126}$ Isotopic species are then selected in the RIBLL2 fragment separator ${ }^{137}$ as shown. In addition to the timing detector shown in the figure, the CSRe is able to employ two additional TOF detectors consisting of $19 \mu \mathrm{g} / \mathrm{cm}^{2}$ carbon foils which generate secondary electrons focused onto a microchannel plate. ${ }^{138}$ These two detectors have been installed in the straight section of the ring opposite the electron cooler (shown in the figure). The nominal circumference of the CSRe is $129 \mathrm{~m}$, and the magnetic rigidity can reach $8.4 \mathrm{Tm} .{ }^{133}$ The use of the dual detectors is to correct for the velocity spread of the particles in the ring. ${ }^{126}$ 
The HIRFL facility has also done a significant amount of work in correcting for magnetic field drift over the course of an experiment, providing for a significant improvement in time and frequency measurements. ${ }^{139}$

\subsubsection{RIKEN Rare RI-Ring}

The Rare RI-Ring (R3) is located in Saitama, Japan. It is a $60 \mathrm{~m}$ circumference ring with a $1 \%$ momentum acceptance. ${ }^{105}$ Heavy beams with energies $\sim 200 \mathrm{MeV} / \mathrm{u}$ are injected, resulting in a revolution time of about $355 \mathrm{~ns}$.

Nuclei are separated by the BigRIPS facility from the primary target between a ring cyclotron and the BigRIPS. This facility was commissioned in 2015. Because the RIKEN Radioactive Ion Beam Factory (RIBF) can produce a very high intensity ${ }^{238} \mathrm{U}$ beam, more exotic nuclear species can be produced at higher intensities. One interesting aspect of the R3 device is that particle ID is performed in the BigRIPS facility, and no identification is necessary within the ring itself. Plans at R3 are to measure nuclear masses in IMS mode with a resolution of $\delta m / m \sim 10^{-6}$.

\subsection{Other Techniques}

In addition to traps, rings, and TOF methods, other systems very similar to those previously mentioned are worth discussing. In particular, multi-reflection TOF (MR-TOF) mass measurement techniques as well as some innovative methods exist. A few additional techniques are described here.

\subsection{1. $M R-T O F$}

With the Multi-reflection time-of-flight method, ions are reflected back and forth between two electrostatic mirrors in a short tube. ${ }^{140}$ This effectively takes a long flight path and "folds" it several times into a short device. The ions are then ejected from the device to be incident onto a timing detector. Several measurements can be made in which the number of reflections is varied, which can provide an indication of the time for one passage of the particle in the device.

As an example, consider the KEK-MRTOF system, located in Japan, has proven to be quite useful for nuclear mass measurements. It is capable of measuring masses with a resolution $\delta m / m \sim 10^{-7}$ at high rates and a measurement cycle of $\approx 30$ ms. ${ }^{141,142}$

A schematic diagram of the setup of the KEK-MRTOF is shown in Figure $10 .{ }^{143}$ An MRTOF spectrometer first traps ions. After this, it injects those ions into a drift chamber in which they are reflected back and forth via electrostatic mirrors. The time spent in the drift tube prior to ejection depends on the oscillation RF frequency of the electrostatic mirrors and the ion mass. The ions are then ejected from the tube where a timing detector is used to provide a measurement of flight time in the tube. The measured velocity resolution can be improved by tuning the 


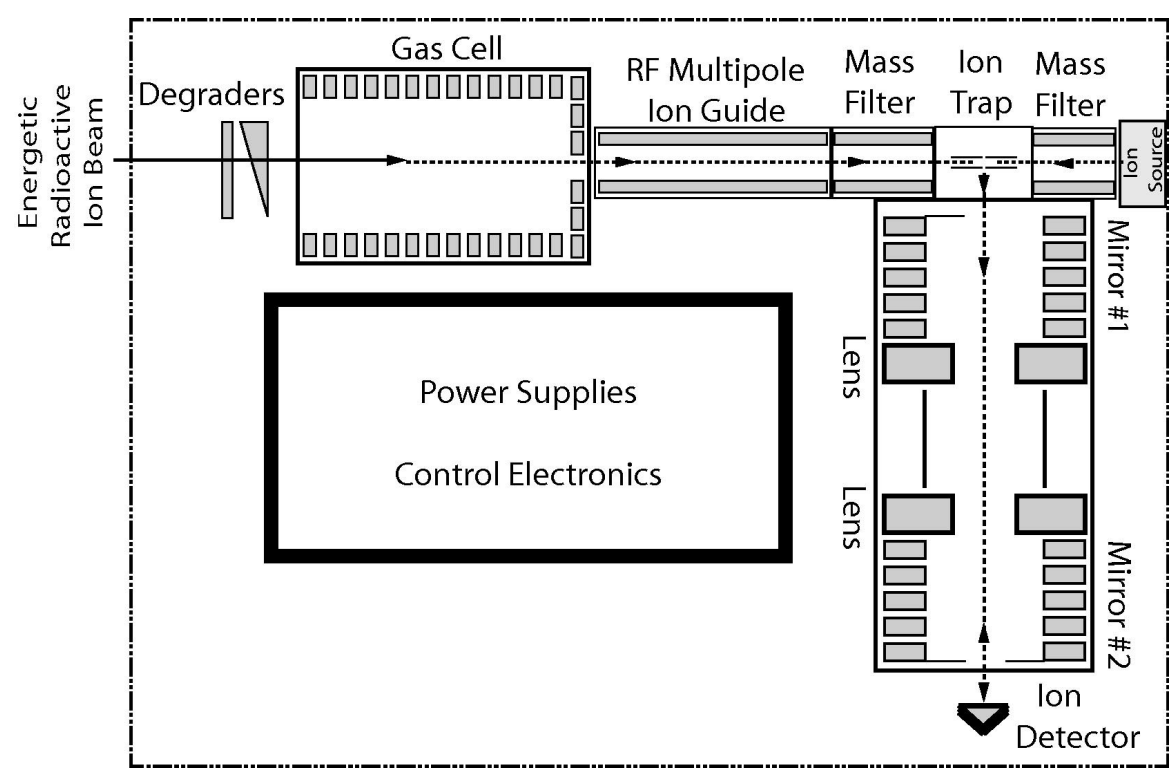

Fig. 10. Schematic diagram of the KEK-MRTOF. ${ }^{143}$ After ions enter the ion trap, they are injected into the spectrometer (right side of the figure). Used with permission. ${ }^{143}$

RF frequency of the electrostatic mirror to increase the ion flight time within the limitations of its lifetime.

The KEK-MRTOF has been used to provide first-time mass measurements of rare-earth nuclei with $204 \leq \mathrm{A} \leq 206 .{ }^{141}$

Other MRTOF systems have been installed at other facilities, including - but not limited to - CERN-ISOLDE, ${ }^{25}$ GANIL, ${ }^{144}$ Notre Dame, ${ }^{145}$ Argonne, ${ }^{146}$ RIKEN $^{147}$ TRIUMF, ${ }^{148}$ and GSI. ${ }^{149}$

\subsubsection{MISTRAL}

The MISTRAL device at ISOLDE is a frequency-based measurement system which is used to measure the masses of nuclei via their cyclotron frequency in a uniform magnetic field. ${ }^{150}$ With this device, ions enter an RF modulator, in which their kinetic energy is determined. After this, they enter a uniform magnetic field, where they travel with a cyclotron motion an simultaneous longitudinal motion prior to extraction. ${ }^{151}$ In order to be successfully extracted, they must undergo a specific number of cyclotron orbits. Otherwise, they are not extracted. Thus, the external 


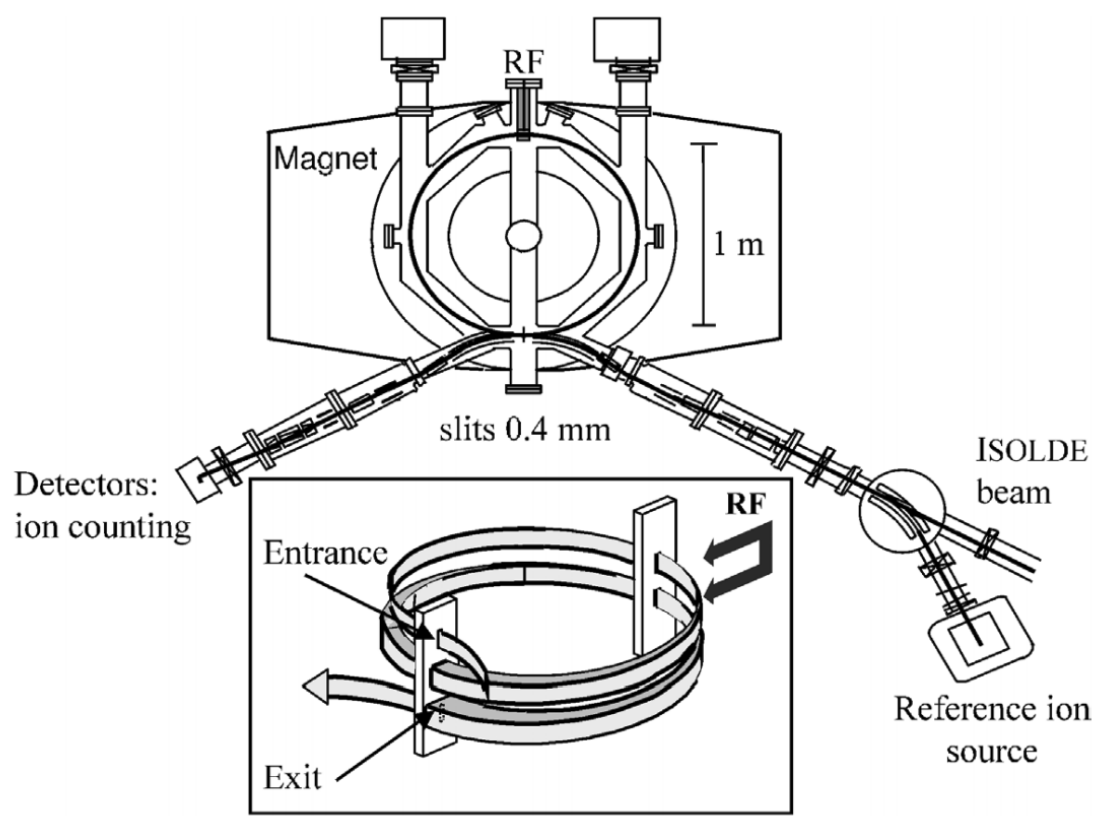

Fig. 11. Top: Drawing of the MISTRAL spectrometer. Bottom: Schematic of a particle path in MISTRAL. ${ }^{152}$ Used with permission. ${ }^{152}$

field is directly related to ion mass. A drawing of the MISTRAL device and a schematic of the path of a particle in MISTRAL is shown in Figure 11. ${ }^{152}$

To date, MISTRAL has been used to measure the masses of light, neutronrich nuclei, including ${ }^{26} \mathrm{Ne},{ }^{26-30} \mathrm{Na},{ }^{29-33} \mathrm{Mg},{ }^{11} \mathrm{Li}$ and ${ }^{11,12} \mathrm{Be}^{150,152-154}$ with a resolution of $\sim 20 \mathrm{keV}$.

\section{Mass Measurement Compilations and Evaluations}

Many existing mass compilations are now supplemented by online resources in various formats which allow for easy access, searches, and analysis. As the field of nuclear physics continues to grow rapidly, with the volume of data growing, these online resources can be extremely useful in searching for updated data as well as for planning future experiments.

Among these online resources are nuclearmasses.org ${ }^{155}$ and the National Nuclear Data Center (NNDC). ${ }^{156}$ The nuclearmasses.org online resources contains a useful interface for comparing multiple datasets as well as up-to-date compilation of new measurements. The NNDC provides an interactive tool for examining the isotopic chart color-coded by multiple properties. In addition, it contains links to 
access other data sets.

The most recent Atomic Mass Evaluation, AME2016, ${ }^{15,16}$ replaces the former AME03. ${ }^{157-159}$ The online volume also provides user-friendly access to the NUBASE2016 tables ${ }^{15,16,160}$ in various formats. This compilation also provides evaluations and evaluation procedures of extrapolated masses and properties. The NUBASE2016 tables can be accessed in electronic form from multiple online resources. ${ }^{161,162}$

In addition to recent mass evaluations, the Atomic Mass Compilation 2012 $(\mathrm{AMC} 12)^{163}$ contains evaluated and experimentally determined masses, which is useful in that the tabulated results indicate the facility from which the results come. Comparisons between AMC12 and AME03 have been made in additional resources. ${ }^{164}$

\section{Future Work}

There is still a significant portion of the table of isotopes yet to be explored. Probing the drip lines and verifying particle stability of nuclei is perhaps the most fundamental nuclear property to probe. Beyond this, perhaps the second-most fundamental property is nuclear masses, though this is not necessarily the easiest to measure. The field of measuring nuclear masses is far from closed, and multiple directions and facilities are being developed.

New opportunities and ideas have arisen in the past few years. Notably, studies of superheavy elements, the discovery of new elements, and new developments in the field of multi-messenger astronomy have made significant progress. These fields, among others, have provided additional impetus to continue to probe the table of isotopes.

From an astrophysical standpoint, nuclear masses are fundamentally important in understanding specific astrophysical processes. For example, as mentioned in the introduction, masses of nuclei along the r-process path are useful in extracting neutron separation energies and shell structure, thus informing further theoretical development.

Multiple facilities are currently being developed or upgraded to address - among other things - the needs of nuclear mass measurement programs. Perhaps the greatest need for new facilities is the need to produce more exotic nuclei with production rates that are accessible with mass measurement programs and techniques. Some of the newest facilities under development are listed here. These are all in various stages of development.

\subsection{FRIB}

The Facility for Rare Isotope Beams (FRIB) is nearing completion with an anticipated commissioning date of around 2022. This facility represents a major reconstruction of the NSCL, described in $\S 2.3 .2$ with the predicted rare isotope production rates shown in Figure 7. 
While the scientific scope of the FRIB facility is broad, its use measuring nuclear masses has not been overlooked. ${ }^{46,165}$ With the expected capabilities of this facility, nuclear mass measurements using the existing LEBIT facility ${ }^{17}$ may be possible. For nuclei beyond the reach of LEBIT, measurements via the $\mathrm{B} \rho$-TOF method are possible. To address this method, the High Rigidity Spectrometer (HRS) is being developed. ${ }^{81}$

\subsection{GSI-FAIR}

The Facility for Antiproton and Ion Research at GSI (GSI-FAIR) began construction in summer 2017. It will use existing facilities at GSI as input stages to the designed accelerator complex. ${ }^{166}$ This facility is expected to provide high-intensity beams covering a range of species, including uranium. ${ }^{167}$

Nuclear mass measurements at the upgraded facility are planned as part of the NuSTAR project. ${ }^{168,169}$ Masses measured at GSI-FAIR will benefit from beam intensities of one to a few orders of magnitude higher than existing GSI intensities. Nuclear masses can be measured with improved techniques including the use of storage rings at GSI. ${ }^{170}$

\section{3. $R A O N$}

In Korea, the Rare isotope Accelerator complex for ON-line experiment (RAON) began construction in 2018 with an estimated completion date in 2021. ${ }^{171}$ This facility is planned to have both isotope separation online (ISOL) and an in-flight fragmentation capabilities.

The vision for the planned facility includes a MR-TOF system to achieve mass resolutions of $\sim 10^{-6}$. The separator is predicted to produce some very neutronrich nuclei. Masses of very exotic nuclei, possibly as neutron-rich as ${ }^{60} \mathrm{Ca}$ may be possible when the facility comes online. ${ }^{171}$

\section{4. $H I A F$}

The High Intensity heavy-ion Accelerator Facility (HIAF) is the planned nextgeneration facility to upgrade the existing HIRFL facility in China. ${ }^{172,173}$ The first beam from HIAF is planned in 2024.

For nuclear mass measurements, the Spectrometer Ring (SRing) is planned, ${ }^{173}$ a $15 \mathrm{Tm}$ ring with a circumference of $273.5 \mathrm{~m}$ and excellent momentum resolution.

\section{Conclusions}

As new online and planned radioactive ion beam facilities push production rates of exotic nuclei to higher values, the field of nuclear mass measurements continues to grow. Production rates of even more exotic nuclei are becoming high enough to make these nuclei accessible by various techniques. While the lifetimes of some of 
the very neutron-rich nuclei are so short that some techniques are excluded, it may still be possible to measure nuclear masses with existing TOF methods (see Figure 2).

TOF methods are currently being accompanied by development of high-rigidity spectrometers, some of which are already in use, such as the SHARAQ spectrometer. ${ }^{98,174}$ Others are currently being planned, such as the FRIB HRS. ${ }^{81}$ These are outfitted with beamline detectors for high-precision determination of particle rigidity, which is necessary for precise event-by-event measurements as well as separation of charge states.

The new facilities and improvements in existing techniques will result in a better understanding of mass models far from stability, evolution of shell structure, and better understanding of astrophysical processes in the next half decade.

Future mass measurements will be useful in informing theoretical developments as well. As computational power improves, the ability for larger basis sets in shell model calculations can result in more precise predictions. As more advanced mass models become available, these can be tested and better constrained with additional measurements. While much of mass measurement experimentation centers on masses of astrophysical interest and more exotic masses, it is still useful to push the limits of known nuclear masses even one or two mass units at a time because many existing mass models disagree significantly outside the regions of known nuclear masses.

\section{References}

1. C. Iliadis, Nuclear Physics of Stars (Wiley-VCH Verlag, 2007).

2. Z. Meisel, S. George, S. Ahn, D. Bazin, B. A. Brown, J. Browne, J. F. Carpino, H. Chung, A. L. Cole, R. H. Cyburt, A. Estradé, M. Famiano, A. Gade, C. Langer, M. Matoš, W. Mittig, F. Montes, D. J. Morrissey, J. Pereira, H. Schatz, J. Schatz, M. Scott, D. Shapira, K. Smith, J. Stevens, W. Tan, O. Tarasov, S. Towers, K. Wimmer, J. R. Winkelbauer, J. Yurkon and R. G. T. Zegers, Physical Review Letters 115 (October 2015) 162501, arXiv:1510.01510 [nucl-ex].

3. Z. Meisel, S. George, S. Ahn, D. Bazin, B. A. Brown, J. Browne, J. F. Carpino, H. Chung, R. H. Cyburt, A. Estradé, M. Famiano, A. Gade, C. Langer, M. Matoš, W. Mittig, F. Montes, D. J. Morrissey, J. Pereira, H. Schatz, J. Schatz, M. Scott, D. Shapira, K. Sieja, K. Smith, J. Stevens, W. Tan, O. Tarasov, S. Towers, K. Wimmer, J. R. Winkelbauer, J. Yurkon and R. G. T. Zegers, Phys. Rev. C 93 (March 2016) 035805, arXiv: 1603.07614 [nucl-ex].

4. A. M. Rogers, M. A. Famiano, W. G. Lynch, M. S. Wallace, F. Amorini, D. Bazin, R. J. Charity, F. Delaunay, R. T. de Souza, J. Elson, A. Gade, D. Galaviz, M.-J. van Goethem, S. Hudan, J. Lee, S. Lobastov, S. Lukyanov, M. Matoš, M. Mocko, H. Schatz, D. Shapira, L. G. Sobotka, M. B. Tsang and G. Verde, Phys. Rev. Lett. 106 (Jun 2011) 252503.

5. B. Pfeiffer, K.-L. Kratz, F.-K. Thielemann and W. Walters, Nuclear Physics A 693 (2001) 282, Radioactive Nuclear Beams.

6. D. Martin, A. Arcones, W. Nazarewicz and E. Olsen, Physical Review Letters 116 (March 2016) 121101, arXiv:1512.03158 [nucl-th].

7. M. R. Mumpower, R. Surman, D.-L. Fang, M. Beard, P. Möller, T. Kawano and 
A. Aprahamian, Phys. Rev. C 92 (September 2015) 035807, arXiv:1505.07789 [nucl-th].

8. D. Atanasov, P. Ascher, K. Blaum, R. B. Cakirli, T. E. Cocolios, S. George, S. Goriely, F. Herfurth, H.-T. Janka, O. Just, M. Kowalska, S. Kreim, D. Kisler, Y. A. Litvinov, D. Lunney, V. Manea, D. Neidherr, M. Rosenbusch, L. Schweikhard, A. Welker, F. Wienholtz, R. N. Wolf and K. Zuber, Phys. Rev. Lett. 115 (Dec 2015) 232501.

9. M. Mumpower, R. Surman, D. L. Fang, M. Beard and A. Aprahamian, Journal of Physics G Nuclear Physics 42 (March 2015) 034027.

10. T. Kajino, W. Aoki, A. Balantekin, R. Diehl, M. Famiano and G. Mathews, Progress in Particle and Nuclear Physics XX (2019).

11. B. H. Sun, Y. A. Litvinov, I. Tanihata and Y. H. Zhang, Frontiers of Physics 10 (August 2015) 1, arXiv:1508.00695 [nucl-ex].

12. M. Matoš, A. Estrade, A. M. Amthor, D. Bazin, A. Becerril, T. Elliot, M. Famiano, A. Gade, D. Galaviz, G. Lorusso, J. Pereira, M. Portillo, A. Rogers, H. Schatz, D. Shapira, E. Smith, A. Stolz and M. Wallace, Acta Physica Polonica B 40 (March 2009) 695.

13. S. Rahaman, V.-V. Elomaa, T. Eronen, U. Hager, J. Hakala, A. Jokinen, A. Kankainen, J. Rissanen, C. Weber and J. Aeystoe, Acta Physica Polonica B 39 (February 2008) 463, arXiv:0801.0487 [nucl-ex].

14. W. Mittig, Nuclear Physics A 553 (1993) 473.

15. W. Huang, G. Audi, M. Wang, F. Kondev, S. Naimi and X. Xu, Chinese Physics C 41 (2017) 03002.

16. M. Wang, G. Audi, F. Kondev, W. Huang, S. Naimi and X. Xu, Chinese Physics C 41 (2017) 03003.

17. R. Ringle, G. Bollen, A. Prinke, J. Savory, P. Schury, S. Schwarz and T. Sun, Nuclear Instruments and Methods in Physics Research Section A: Accelerators, Spectrometers, Detectors and Associated Equipment 604 (2009) 536.

18. J. Dilling, K. Blaum, M. Brodeur and S. Eliseev, Annual Review of Nuclear and Particle Science 68 (October 2018) annurev.

19. L. S. Brown and G. Gabrielse, Rev. Mod. Phys. 58 (Jan 1986) 233.

20. G. Savard, R. C. Barber, C. Boudreau, F. Buchinger, J. Caggiano, J. Clark, J. E. Crawford, H. Fukutani, S. Gulick, J. C. Hardy, A. Heinz, J. K. P. Lee, R. B. Moore, K. S. Sharma, J. Schwartz, D. Seweryniak, G. D. Sprouse and J. Vaz, Hyperfine Interactions 132 (January 2001) 221.

21. J. C. Wang, G. Savard, K. S. Sharma, J. A. Clark, Z. Zhou, A. F. Levand, C. Boudreau, F. Buchinger, J. E. Crawford, J. P. Greene, S. Gulick, J. K. P. Lee, G. D. Sprouse, W. Trimble, J. Vaz and B. Z. Zabransky, Nuclear Physics A $\mathbf{7 4 6}$ (December 2004) 651.

22. J. Van Schelt, D. Lascar, G. Savard, J. A. Clark, S. Caldwell, A. Chaudhuri, J. Fallis, J. P. Greene, A. F. Levand, G. Li, K. S. Sharma, M. G. Sternberg, T. Sun and B. J. Zabransky, Phys. Rev. C 85 (April 2012) 045805, arXiv:1203.4470 [nucl-ex].

23. M. Mukherjee, D. Beck, K. Blaum, G. Bollen, J. Dilling, S. George, F. Herfurth, A. Herlert, A. Kellerbauer, H. J. Kluge, S. Schwarz, L. Schweikhard and C. Yazidjian, The European Physical Journal A 35 (Jan 2008) 1.

24. R. N. Wolf, F. Wienholtz, D. Atanasov, D. Beck, K. Blaum, C. Borgmann, F. Herfurth, M. Kowalska, S. Kreim, Y. A. Litvinov, D. Lunney, V. Manea, D. Neidherr, M. Rosenbusch, L. Schweikhard, J. Stanja and Z. Zuber, International Journal of Mass Spectrometry 349 (September 2013) 123.

25. R. Wolf, D. Atanasov, K. Blaum, S. Kreim, D. Lunney, V. Manea, M. Rosenbusch, 
L. Schweikhard, A. Welker, F. Wienholtz and K. Zuber, Nuclear Instruments and Methods in Physics Research Section B: Beam Interactions with Materials and Atoms 376 (2016) 275 , Proceedings of the XVIIth International Conference on Electromagnetic Isotope Separators and Related Topics (EMIS2015), Grand Rapids, MI, U.S.A., 11-15 May 2015.

26. F. Herfurth, G. Audi, D. Beck, K. Blaum, G. Bollen, P. Delahaye, S. George, C. Guénaut, A. Herlert, A. Kellerbauer, H. J. Kluge, D. Lunney, M. Mukherjee, S. Rahaman, S. Schwarz, L. Schweikhard, C. Weber and C. Yazidjian, The European Physical Journal A - Hadrons and Nuclei 25 (Sep 2005) 17.

27. S. Baruah, G. Audi, K. Blaum, M. Dworschak, S. George, C. Guénaut, U. Hager, F. Herfurth, A. Herlert, A. Kellerbauer, H.-J. Kluge, D. Lunney, H. Schatz, L. Schweikhard and C. Yazidjian, Physical Review Letters 101 (December 2008) 262501, arXiv:0811.2294 [nucl-ex].

28. J. Hakala, J. Dobaczewski, D. Gorelov, T. Eronen, A. Jokinen, A. Kankainen, V. S. Kolhinen, M. Kortelainen, I. D. Moore, H. Penttilä, S. Rinta-Antila, J. Rissanen, A. Saastamoinen, V. Sonnenschein and J. Äystö, Phys. Rev. Lett. 109 (Jul 2012) 032501.

29. J. Äystö, Precision mass measurements of neutron-rich nuclei between $\mathrm{N}=50$ and 82, in Journal of Physics Conference Series, , Journal of Physics Conference Series Vol. 420 (March 2013), p. 012045. arXiv:1210.6567 [nucl-ex].

30. A. Kankainen, Hyperfine Interactions 199 (July 2011) 221.

31. S. Rahaman, V.-V. Elomaa, T. Eronen, U. Hager, J. Hakala, A. Jokinen, A. Kankainen, I. D. Moore, H. Penttilä, S. Rinta-Antila, J. Rissanen, A. Saastamoinen, T. Sonoda, C. Weber and J. Ńystö, European Physical Journal Special Topics 150 (November 2007) 349, nucl-ex/0703018.

32. V.-V. Elomaa, T. Eronen, J. Hakala, A. Jokinen, A. Kankainen, I. Moore, S. Rahaman, J. Rissanen, C. Weber and J. yst, Nuclear Instruments and Methods in Physics Research Section A: Accelerators, Spectrometers, Detectors and Associated Equipment 612 (2009) 97 .

33. M. Vilen, J. M. Kelly, A. Kankainen, M. Brodeur, A. Aprahamian, L. Canete, T. Eronen, A. Jokinen, T. Kuta, I. D. Moore, M. R. Mumpower, D. A. Nesterenko, H. Penttilä, I. Pohjalainen, W. S. Porter, S. Rinta-Antila, R. Surman, A. Voss and J. ńystö, Phys. Rev. Lett. 120 (Jun 2018) 262701, arXiv:1801.08940 [nucl-ex].

34. S. Rahaman, J. Hakala, V. V. Elomaa, T. Eronen, U. Hager, A. Jokinen, A. Kankainen, I. D. Moore, H. Penttilä, S. Rinta-Antila, J. Rissanen, A. Saastamoinen, C. Weber and J. Äystö, The European Physical Journal A 34 (Oct 2007) 5 .

35. J. Hakala, S. Rahaman, V.-V. Elomaa, T. Eronen, U. Hager, A. Jokinen, A. Kankainen, I. D. Moore, H. Penttilä, S. Rinta-Antila, J. Rissanen, A. Saastamoinen, T. Sonoda, C. Weber and J. Äystö, Phys. Rev. Lett. 101 (Jul 2008) 052502.

36. S. Rahaman, U. Hager, V. V. Elomaa, T. Eronen, J. Hakala, A. Jokinen, A. Kankainen, P. Karvonen, I. D. Moore, H. Penttilä, S. Rinta-Antila, J. Rissanen, A. Saastamoinen, T. Sonoda and J. Äystö, The European Physical Journal A 32 (Apr 2007) 87.

37. U. Hager, T. Eronen, J. Hakala, A. Jokinen, V. S. Kolhinen, S. Kopecky, I. Moore, A. Nieminen, M. Oinonen, S. Rinta-Antila, J. Szerypo and J. Äystö, Phys. Rev. Lett. 96 (February 2006) 042504.

38. U. Hager, V. V. Elomaa, T. Eronen, J. Hakala, A. Jokinen, A. Kankainen, S. Rahaman, S. Rinta- Antila, A. Saastamoinen, T. Sonoda and J. Äystö, Phys. Rev. C 75 (June 2007) 064302. 
39. U. Hager, A. Jokinen, V. V. Elomaa, T. Eronen, J. Hakala, A. Kankainen, S. Rahaman, J. Rissanen, I. D. Moore, S. Rinta-Antila, A. Saastamoinen, T. Sonoda and J. Äystö, Nucl. Phys. A $\mathbf{7 9 3}$ (September 2007) 20.

40. J. Hakala, R. Rodríguez-Guzmán, V. V. Elomaa, T. Eronen, A. Jokinen, V. S. Kolhinen, I. D. Moore, H. Penttilä, M. Reponen, J. Rissanen, A. Saastamoinen and J. Äystö, European Physical Journal A 47 (October 2011) 129.

41. J. S. E. Wieslander, J. Suhonen, T. Eronen, M. Hult, V. V. Elomaa, A. Jokinen, G. Marissens, M. Misiaszek, M. T. Mustonen, S. Rahaman, C. Weber and J. Äystö, Phys. Rev. Lett. 103 (September 2009) 122501.

42. S. Rinta-Antila, T. Eronen, V. V. Elomaa, U. Hager, J. Hakala, A. Jokinen, P. Karvonen, H. Penttilä, J. Rissanen, T. Sonoda, A. Saastamoinen and J. Äystö, European Physical Journal A 31 (January 2007) 1.

43. R. Ringle, S. Schwarz and G. Bollen, International Journal of Mass Spectrometry 349 (September 2013) 87.

44. S. Schwarz, G. Bollen, S. Chouhan, J. Das, M. Green, C. Magsig, D. Morrissey, J. Ottarson, C. Sumithrarachchi, A. Villari and A. Zeller, Nuclear Instruments and Methods in Physics Research Section B: Beam Interactions with Materials and Atoms 376 (2016) 256 , Proceedings of the XVIIth International Conference on Electromagnetic Isotope Separators and Related Topics (EMIS2015), Grand Rapids, MI, U.S.A., 11-15 May 2015.

45. S. Schwarz, G. Bollen, R. Ringle, J. Savory and P. Schury, Nuclear Instruments and Methods in Physics Research Section A: Accelerators, Spectrometers, Detectors and Associated Equipment 816 (2016) 131.

46. A. Gade and B. Sherrill, Physica Scripta 91 (2016) 053003.

47. J. Schönfelder, D. Ackermann, H. Backe, G. Bollen, J. Dilling, A. Dretzke, O. Engels, J. Estermann, D. Habs, S. Hofmann, F. Hessberger, H.-J. Kluge, W. Lauth, W. Ludolphs, M. Maier, G. Marx, R. Moore, W. Quint, D. Rodriguez, M. Sewtz, G. Sikler, C. Toader and C. Weber, Nuclear Physics A 701 (2002) 579 , 5th International Conference on Radioactive Nuclear Beams.

48. M. Block, D. Ackermann, D. Beck, K. Blaum, M. Breitenfeldt, A. Chauduri, A. Doemer, S. Eliseev, D. Habs, S. Heinz, F. Herfurth, F. P. Heßberger, S. Hofmann, H. Geissel, H. J. Kluge, V. Kolhinen, G. Marx, J. B. Neumayr, M. Mukherjee, M. Petrick, W. Plass, W. Quint, S. Rahaman, C. Rauth, D. Rodrıguez, C. Scheidenberger, L. Schweikhard, M. Suhonen, P. G. Thirolf, Z. Wang and C. Weber, The European Physical Journal A - Hadrons and Nuclei 25 (Sep 2005) 49.

49. M. Block, D. Ackermann, K. Blaum, A. Chaudhuri, Z. Di, S. Eliseev, R. Ferrer, D. Habs, F. Herfurth, F. P. Heßberger, S. Hofmann, H.-J. Kluge, G. Maero, A. Martín, G. Marx, M. Mazzocco, M. Mukherjee, J. B. Neumayr, W. R. Plaß, W. Quint, S. Rahaman, C. Rauth, D. Rodríguez, C. Scheidenberger, L. Schweikhard, P. G. Thirolf, G. Vorobjev and C. Weber, Hyperfine Interactions 173 (Nov 2006) 133.

50. D. Lascar, A. A. Kwiatkowski, M. Alanssari, U. Chowdhury, J. Even, A. Finlay, A. T. Gallant, M. Good, R. Klawitter, B. Kootte, T. Li, K. G. Leach, A. Lennarz, E. Leistenschneider, A. J. Mayer, B. E. Schultz, R. Schupp, D. A. Short, C. Andreoiu, J. Dilling and G. Gwinner, Nuclear Instruments and Methods in Physics Research B 376 (June 2016) 292, arXiv:1508.06693 [physics.ins-det].

51. J. Dilling, R. Baartman, P. Bricault, M. Brodeur, L. Blomeley, F. Buchinger, J. Crawford, J. C. Lpez-Urrutia, P. Delheij, M. Froese, G. Gwinner, Z. Ke, J. Lee, R. Moore, V. Ryjkov, G. Sikler, M. Smith, J. Ullich and J. Vaz, International Journal of Mass Spectrometry 251 (2006) 198 , ULTRA-ACCURATE MASS SPECTROME- 
TRY AND RELATED TOPICS Dedicated to H.-J. Kluge on the occasion of his 65th birthday anniversary.

52. P. Delheij, L. Blomeley, M. Froese, G. Gwinner, V. Ryjkov, M. Smith and J. Dilling, Hyperfine Interactions 173 (Nov 2006) 123.

53. M. Froese, C. Champagne, J. R. Crespo López-Urrutia, S. Epp, G. Gwinner, A. Lapierre, J. Pfister, G. Sikler, J. Ullrich and J. Dilling, Hyperfine Interactions 173 (Nov 2006) 85.

54. A. A. Kwiatkowski, J. Dilling, C. Andreoiu, T. Brunner, A. Chaudhuri, U. Chowdhury, P. Delheij, S. Ettenauer, D. Frekers, A. T. Gallant, A. Grossheim, G. Gwinner, A. Lennarz, E. Mané, M. R. Pearson, B. E. Schultz, M. C. Simon and V. V. Simon, Precision Penning Trap Mass Measurements for Nuclear Structure at Triumf, in Capture Gamma-Ray Spectroscopy and Related Topics, eds. P. E. Garrett and et al. (March 2013), pp. 409-414.

55. J. Dilling, P. Bricault, M. Smith and H.-J. Kluge, Nuclear Instruments and Methods in Physics Research Section B: Beam Interactions with Materials and Atoms 204 (2003) 492 , 14th International Conference on Electromagnetic Isotope Separators and Techniques Related to their Applications.

56. R. Klawitter, A. Bader, M. Brodeur, U. Chowdhury, A. Chaudhuri, J. Fallis, A. T. Gallant, A. Grossheim, A. A. Kwiatkowski, D. Lascar, K. G. Leach, A. Lennarz, T. D. Macdonald, J. Pearkes, S. Seeraji, M. C. Simon, V. V. Simon, B. E. Schultz and J. Dilling, Phys. Rev. C 93 (Apr 2016) 045807.

57. D. Lascar, R. Klawitter, C. Babcock, E. Leistenschneider, S. R. Stroberg, B. R. Barquest, A. Finlay, M. Foster, A. T. Gallant, P. Hunt, J. Kelly, B. Kootte, Y. Lan, S. F. Paul, M. L. Phan, M. P. Reiter, B. Schultz, D. Short, J. Simonis, C. Andreoiu, M. Brodeur, I. Dillmann, G. Gwinner, J. D. Holt, A. A. Kwiatkowski, K. G. Leach and J. Dilling, Phys. Rev. C 96 (Oct 2017) 044323.

58. M. Smith, M. Brodeur, T. Brunner, S. Ettenauer, A. Lapierre, R. Ringle, V. L. Ryjkov, F. Ames, P. Bricault, G. W. F. Drake, P. Delheij, D. Lunney, F. Sarazin and J. Dilling, Phys. Rev. Lett. 101 (Nov 2008) 202501.

59. B. J. Mount, M. Redshaw and E. G. Myers, Physical Review A 82 (Oct 2010) 042513.

60. I. Bergstrm, C. Carlberg, T. Fritioff, G. Douysset, J. Schnfelder and R. Schuch, Nuclear Instruments and Methods in Physics Research Section A: Accelerators, Spectrometers, Detectors and Associated Equipment 487 (2002) 618 .

61. J. Cornejo and D. Rodrguez, Nuclear Instruments and Methods in Physics Research Section B: Beam Interactions with Materials and Atoms 376 (2016) 288, Proceedings of the XVIIth International Conference on Electromagnetic Isotope Separators and Related Topics (EMIS2015), Grand Rapids, MI, U.S.A., 11-15 May 2015.

62. P. Ascher, B. Blank, K. Blaum, P. Dupré, M. Gerbaux, S. Grévy, H. Guérin, M. Heck, D. Lunney, S. Naimi and A. de Roubin, PIPERADE: A Penning-trap isobar separator for the DESIR low-energy facility of SPIRAL2, in European Physical Journal Web of Conferences, (Mar 2014), p. Di.

63. B. Delauré, M. Beck, V. V. Golovko, V. Kozlov, T. Phalet, P. Schuurmans, N. Severijns, B. Vereecke, S. Versyck, D. Beck, W. Quint, F. Ames, K. Reisinger, O. Forstner, J. Deutsch, G. Bollen and S. Schwarz, Witch: A penning trap retardation spectrometer combination for precision studies of the weak interaction, in Atomic Physics at Accelerators: Stored Particles and Fundamental Physics, eds. H. Knudsen, J. U. Andersen and H.-J. Kluge (Springer Netherlands, Dordrecht, 2003), pp. 91-95.

64. F. Ames, G. Bollen, G. Huber and P. Schmidt, REXTRAP, an ion buncher for REXISOLDE, in American Institute of Physics Conference Series, eds. B. M. Sherill, D. J. Morrissey and C. N. Davids (Dec 1998), pp. 927-932. 
65. C. Smorra, T. Beyer, K. Blaum, M. Block, K. Eberhardt, M. Eibach, F. Herfurth, J. Ketelaer, K. Knuth, W. Nörtershäuser and S. Nagy, High-Precision Mass Measurements At TRIGA-TRAP, in American Institute of Physics Conference Series, eds. Y. E. Penionzhkevich and S. M. Lukyanov, American Institute of Physics Conference Series, Vol. 1224 (Apr 2010), pp. 544-551.

66. L. Schweikhard, S. Krückeberg, K. Lützenkirchen and C. Walther, European Physical Journal D 9 (Jan 1999) 15.

67. Z. Andjelkovic, W. Barth, G. Birkl, I. Blank, K. Blaum, N. P. M. Brantjes, E. Bodewits, A. Bräuning-Demian, D. Church, L. Dahl, S. Götz, F. Herfurth, R. Hoekstra, O. Kester, H. J. Kluge, S. Koszudowski, C. Kozhuharov, G. Maero, W. Nörtershäuser, J. Pfister, W. Quint, U. Ratzinger, A. Schempp, D. Segal, A. Sokolov, T. Stöhlker, R. C. Thompson, J. Ullrich, V. Varentsov, M. Vogel, G. Vorobjev, A. Warczak, M. Weidemüller, C. Weinheimer and D. F. A. Winters, HITRAP - a facility for experiments on heavy highly charged ions and on antiprotons, in Journal of Physics Conference Series, (Nov 2009), p. 142007.

68. J. Repp, C. Böhm, J. R. Crespo López-Urrutia, A. Dörr, S. Eliseev, S. George, M. Goncharov, Y. N. Novikov, C. Roux, S. Sturm, S. Ulmer and K. Blaum, Applied Physics B: Lasers and Optics 107 (Jun 2012) 983, arXiv:1110.2919 [physics.atom-ph].

69. C. Weber, R. Meißner, P. Müller and P. G. Thirolf, Hyperfine Interactions 227 (Jun 2014) 247.

70. W. Huang, Y. Tian, J. Wang, Y. Sun, Y. Wang, Y. Wang, J. Zhao, W. Wu, L. Ma, Y. He, H. Xu and G. Xiao, Nuclear Instruments and Methods in Physics Research Section B: Beam Interactions with Materials and Atoms 317 (2013) 528 , XVIth International Conference on ElectroMagnetic Isotope Separators and Techniques Related to their Applications, December 27, 2012 at Matsue, Japan.

71. F. Heiße, F. Köhler-Langes, S. Rau, J. Hou, S. Junck, A. Kracke, A. Mooser, W. Quint, S. Ulmer, G. Werth, K. Blaum and S. Sturm, Phys. Rev. Lett. 119 (Jul 2017) 033001.

72. A. Estradé, M. Matoš, M. A. Amthor, D. Bazin, A. D. Becerril, T. J. Elliot, A. Gade, D. Galaviz, G. Lorusso, J. Pereira, M. Portillo, A. Rogers, H. Schatz, D. Shapira, E. Smith, A. Stolz and M. S. Wallace, TOF-B $\rho$ Mass Measurement of Neutron Rich Nuclei at the NSCL, in VII LATIN American Symposium on Nuclear Physics and Applications, eds. R. Alarcon, P. L. Cole, C. Djalali and F. Umeres, American Institute of Physics Conference Series, Vol. 947 (October 2007), pp. 383-386.

73. M. Matoš, A. Estrade, M. Amthor, A. Aprahamian, D. Bazin, A. Becerril, T. Elliot, D. Galaviz, A. Gade, S. Gupta, G. Lorusso, F. Montes, J. Pereira, M. Portillo, A. M. Rogers, H. Schatz, D. Shapira, E. Smith, A. Stolz and M. Wallace, Journal of Physics G Nuclear Physics 35 (January 2008) 014045, arXiv:0707.0119 [nucl-ex].

74. D. J. Morrissey, B. M. Sherrill, M. Steiner, A. Stolz and I. Wiedenhoever, Nuclear Instruments and Methods in Physics Research B 204 (May 2003) 90.

75. D. Bazin, J. A. Caggiano, B. M. Sherrill, J. Yurkon and A. Zeller, Nuclear Instruments and Methods in Physics Research B 204 (May 2003) 629.

76. D. Shapira, T. Lewis and L. Hulett, Nuclear Instruments and Methods in Physics Research Section A: Accelerators, Spectrometers, Detectors and Associated Equipment 454 (2000) 409 .

77. A. Rogers, A. Sanetullaev, W. Lynch, M. Tsang, J. Lee, D. Bazin, D. Coupland, V. Henzl, D. Henzlova, M. Kilburn, M. Wallace, M. Youngs, F. Delaunay, M. Famiano, D. Shapira, K. Jones, K. Schmitt and Z. Sun, Nuclear Instruments and Methods in Physics Research Section A: Accelerators, Spectrometers, Detectors and Associ- 
ated Equipment 795 (2015) 325 .

78. A. Estrade, M. Mato, H. Schatz, A. Amthor, D. Bazin, M. Beard, E. Brown, A. Becerril, T. Elliot, A. Gade, D. Galaviz, S. Gupta, W. Hix, R. Lau, G. Lorusso, P. Mller, J. Pereira, M. Portillo, A. Rogers and M. Wiescher, Proceedings of Science 100 (01 2010) 221.

79. T. Baumann, M. Hausmann, B. Sherrill and O. Tarasov, Nuclear Instruments and Methods in Physics Research Section B: Beam Interactions with Materials and Atoms 376 (2016) 33 , Proceedings of the XVIIth International Conference on Electromagnetic Isotope Separators and Related Topics (EMIS2015), Grand Rapids, MI, U.S.A., 11-15 May 2015.

80. R. Zegers and A. Gade, A high rigidity spectrometer for frib http://hrs.lbl.gov/science.

81. T. Baumann, Nuclear Instruments and Methods in Physics Research Section B: Beam Interactions with Materials and Atoms 376 (2016) 162 , Proceedings of the XVIIth International Conference on Electromagnetic Isotope Separators and Related Topics (EMIS2015), Grand Rapids, MI, U.S.A., 11-15 May 2015.

82. H. Schatz, International Journal of Mass Spectrometry 349 (September 2013) 181.

83. A. Estradé, M. Matoš, H. Schatz, A. M. Amthor, D. Bazin, M. Beard, A. Becerril, E. F. Brown, R. Cyburt, T. Elliot, A. Gade, D. Galaviz, S. George, S. S. Gupta, W. R. Hix, R. Lau, G. Lorusso, P. Möller, J. Pereira, M. Portillo, A. M. Rogers, D. Shapira, E. Smith, A. Stolz, M. Wallace and M. Wiescher, Physical Review Letters 107 (October 2011) 172503, arXiv:1109.5200 [nucl-ex].

84. M. Matoš, A. Estradé, H. Schatz, D. Bazin, M. Famiano, A. Gade, S. George, W. G. Lynch, Z. Meisel, M. Portillo, A. Rogers, D. Shapira, A. Stolz, M. Wallace and J. Yurkon, Nuclear Instruments and Methods in Physics Research A 696 (December 2012) 171

85. H. Savajols, Hyperfine Interactions 132 (January 2001) 243.

86. H. Savajols, The speg mass measurement program at ganil, in Atomic Physics at Accelerators: Mass Spectrometry: Proceedings of the APAC 2000, held in Cargèse, France, 19-23 September 2000, eds. D. Lunney, G. Audi and H.-J. Kluge (Springer Netherlands, Dordrecht, 2001), Dordrecht, pp. 245-254.

87. M. B. Gómez Hornillos, M. Chartier, W. Mittig, B. Blank, F. Chautard, C. E. Demonchy, A. Gillibert, B. Jacquot, B. Jurado, N. Lecesne, A. Lépine-Szily, N. A. Orr, P. Roussel-Chomaz, H. Savajols and A. C. C. Villari, Journal of Physics G Nuclear Physics 31 (October 2005) S1869.

88. F. Sarazin, H. Savajols, W. Mittig, F. Nowacki, N. A. Orr, Z. Ren, P. RousselChomaz, G. Auger, D. Baiborodin, A. V. Belozyorov, C. Borcea, E. Caurier, Z. Dlouhý, A. Gillibert, A. S. Lalleman, M. Lewitowicz, S. M. Lukyanov, F. de Oliveira, Y. E. Penionzhkevich, D. Ridikas, O. Tarasov, H. Sakuraï and A. de Vismes, Hyperfine Interactions 132 (Jan 2001) 147.

89. A. Gillibert, L. Bianchi, A. Cunsolo, B. Fernandez, A. Foti, J. Gastebois, C. Gregoire, W. Mittig, A. Peghaire, Y. Schutz and C. Stephan, Physics Letters B 176 (1986) 317 .

90. A. Gillibert, W. Mittig, L. Bianchi, A. Cunsolo, B. Fernandez, A. Foti, J. Gastebois, C. Grgoire, Y. Schutz and C. Stephan, Physics Letters B 192 (1987) 39 .

91. N. Orr, W. Mittig, L. Fifield, M. Lewitowicz, E. Plagnol, Y. Schutz, Z. W. Long, L. Bianchi, A. Gillibert, A. Belozyorov, S. Lukyanov, Y. Penionzhkevich, A. Villari, A. Cunsolo, A. Foti, G. Audi, C. Stephan and L. Tassan-Got, Physics Letters B 258 (1991) 29 .

92. F. Sarazin, H. Savajols, W. Mittig, F. Nowacki, N. A. Orr, Z. Ren, P. Roussel- 
Chomaz, G. Auger, D. Baiborodin, A. V. Belozyorov, C. Borcea, E. Caurier, Z. Dlouhý, A. Gillibert, A. S. Lalleman, M. Lewitowicz, S. M. Lukyanov, F. de Oliveira, Y. E. Penionzhkevich, D. Ridikas, H. Sakuraï, O. Tarasov and A. de Vismes, Phys. Rev. Lett. 84 (May 2000) 5062.

93. H. Savajols, B. Jurado, W. Mittig, D. Baiborodin, W. Catford, M. Chartier, C. E. Demonchy, Z. Dlouhy, A. Gillibert, L. Giot, A. Khouaja, A. Lépine-Szily S. Lukyanov, J. Mrazek, N. Orr, Y. Penionzhkevich, S. Pita, M. Rousseau, P. Roussel-Chomaz and A. C. C. Villari, The European Physical Journal A - Hadrons and Nuclei 25 (Sep 2005) 23.

94. B. Jurado, H. Savajols, W. Mittig, N. Orr, P. Roussel-Chomaz, D. Baiborodin, W. Catford, M. Chartier, C. Demonchy, Z. Dlouh, A. Gillibert, L. Giot, A. Khouaja, A. Lpine-Szily, S. Lukyanov, J. Mrazek, Y. Penionzhkevich, S. Pita, M. Rousseau and A. Villari, Physics Letters B 649 (2007) 43 .

95. L. Gaudefroy, W. Mittig, N. A. Orr, S. Varet, M. Chartier, P. Roussel-Chomaz, J. P. Ebran, B. Fernández-Domínguez, G. Frémont, P. Gangnant, A. Gillibert, S. Grévy, J. F. Libin, V. A. Maslov, S. Paschalis, B. Pietras, Y.-E. Penionzhkevich, C. Spitaels and A. C. C. Villari, Phys. Rev. Lett. 109 (Nov 2012) 202503.

96. T. Uesaka, S. Shimoura, H. Sakai, G. Berg, K. Nakanishi, Y. Sasamoto, A. Saito, S. Michimasa, T. Kawabata and T. Kubo, Nuclear Instruments and Methods in Physics Research Section B: Beam Interactions with Materials and Atoms 266 (2008) 4218 , Proceedings of the XVth International Conference on Electromagnetic Isotope Separators and Techniques Related to their Applications.

97. T. Uesaka, S. Shimoura and H. Sakai, Progress of Theoretical and Experimental Physics 2012 (2012) $03 \mathrm{C} 007$.

98. S. Michimasa, M. Takaki, Y. Sasamoto, M. Dozono, T. Nishi, T. Kawabata, S. Ota, H. Baba, T. Baba, T. Fujii, S. Go, S. Kawase, Y. Kikuchi, K. Kisamori, M. Kobayashi, Y. Kubota, C. Lee, H. Matsubara, K. Miki, H. Miya, S. Noji, H. Tokieda, M. Tsumura, K. Yako, R. Yokoyama, H. Takeda, Y. Yanagisawa, T. Kubo, N. Inabe, N. Fukuda, D. Kameda, H. Suzuki, Y. Shimizu, H. Sato, T. Ichihara, A. Stolz, R. Zegers, H. Sakai, T. Uesaka and S. Shimoura, Nuclear Instruments and Methods in Physics Research Section B: Beam Interactions with Materials and Atoms 317 (2013) 305 , XVIth International Conference on ElectroMagnetic Isotope Separators and Techniques Related to their Applications, December 27, 2012 at Matsue, Japan.

99. S. Michimasa, M. Takaki, M. Dozono, S. Go, H. Baba, E. Ideguchi, K. Kisamori, H. Matsubara, H. Miya, S. Ota, H. Sakai, S. Shimoura, A. Stolz, T. Tang, H. Tokieda, T. Uesaka and R. Zegers, Nuclear Instruments and Methods in Physics Research Section B: Beam Interactions with Materials and Atoms 317 (2013) 710 , XVIth International Conference on ElectroMagnetic Isotope Separators and Techniques Related to their Applications, December 27, 2012 at Matsue, Japan.

100. J. Xia, W. Zhan, B. Wei, Y. Yuan, M. Song, W. Zhang, X. Yang, P. Yuan, D. Gao, H. Zhao, X. Yang, G. Xiao, K. Man, J. Dang, X. Cai, Y. Wang, J. Tang, W. Qiao, Y. Rao, Y. He, L. Mao and Z. Zhou, Nuclear Instruments and Methods in Physics Research Section A: Accelerators, Spectrometers, Detectors and Associated Equipment 488 (2002) 11.

101. J. Zhao, B. Sun, I. Tanihata, S. Terashima, L. Zhu, A. Enomoto, D. Nagae, T. Nishimura, S. Omika, A. Ozawa, Y. Takeuchi and T. Yamaguchi, Nuclear Instruments and Methods in Physics Research Section A: Accelerators, Spectrometers, Detectors and Associated Equipment 823 (2016) 41.

102. J. Wouters, D. Vieira, H. Wollnik, H. Enge, S. Kowalski and K. Brown, Nuclear In- 
struments and Methods in Physics Research Section A: Accelerators, Spectrometers, Detectors and Associated Equipment 240 (1985) 77.

103. D. J. Vieira, J. M. Wouters, K. Vaziri, R. H. Kraus, H. Wollnik, G. W. Butler, F. K. Wohn and A. H. Wapstra, Phys. Rev. Lett. 57 (Dec 1986) 3253.

104. J. M. Wouters, R. H. Kraus, D. J. Vieira, G. W. Butler and K. E. G. Löbner, Zeitschrift für Physik A Atomic Nuclei 331 (Sep 1988) 229.

105. A. Ozawa, T. Uesaka and M. Wakasugi, Progress of Theoretical and Experimental Physics 2012 (2012) 03C009.

106. A. Ozawa, Rare-RI ring for mass measurements at RIBF, in American Institute of Physics Conference Series, eds. S. Jeong, N. Imai, H. Miyatake and T. Kajino, American Institute of Physics Conference Series, Vol. 1594 (May 2014), pp. 365-369.

107. F. Nolden, C. Dimopoulou, A. Dolinskii and M. Steck, Nuclear Instruments and Methods in Physics Research Section B: Beam Interactions with Materials and Atoms 266 (2008) 4569 , Proceedings of the XVth International Conference on Electromagnetic Isotope Separators and Techniques Related to their Applications.

108. B. Franzke, H. Geissel and G. Mnzenberg, Mass Spectrometry Reviews 27 (2008) 428.

109. M. Hausmann, F. Attallah, K. Beckert, F. Bosch, A. Dolinskiy, H. Eickhoff, M. Falch, B. Franczak, B. Franzke, H. Geissel, T. Kerscher, O. Klepper, H. J. Kluge, C. Kozhuharov, K. E. G. Löbner, G. Münzenberg, F. Nolden, Y. N. Novikov, T. Radon, H. Schatz, C. Scheidenberger, J. Stadlmann, M. Steck, T. Winkler and H. Wollnik, Nuclear Instruments and Methods in Physics Research A 446 (May 2000) 569 .

110. H. Wollnik, K. Beckert, T. Beha, F. Bosch, A. Dolinskii, H. Eickhoff, M. Falch, B. Franzke, Y. Fujita, H. Geissel, M. Hausmann, T. Horvath, H. Irnich, H. C. Jung, T. Kerscher, O. Klepper, C. Kozhuharov, G. Kraus, K. E. G. Löbner, G. Münzenberg, F. Nickel, F. Nolden, Y. Novikov, T. Radon, H. Reich, C. Scheidenberger, B. Schlitt, W. Schwab, A. Schwinn, M. Steck, K. Sümmerer, M. Theiss and T. Winkler, Nuclear Physics A 626 (February 1997) 327.

111. F. Caspers and D. Möhl, 6.10 beam cooling: Datasheet from landolt-börnstein group i elementary particles, nuclei and atoms · volume 21c: "accelerators and colliders" in springermaterials (https://dx.doi.org/10.1007/978-3-642-23053-0_15) accessed 2019-03-16.

112. B. Schlitt, K. Beckert, F. Bosch, H. Eickhoff, B. Franzke, Y. Fujita, H. Geissel, M. Hausmann, H. Irnich, O. Klepper, H.-J. Kluge, C. Kozhuharov, G. Kraus, G. Mnzenberg, F. Nickel, F. Nolden, Z. Patyk, T. Radon, H. Reich, C. Scheidenberger, W. Schwab, M. Steck, K. Smmerer, T. Winkler, T. Beha, M. Falch, T. Kerscher, K. Lbner, H. Jung, H. Wollnik and Y. Novikov, Nuclear Physics A 626 (1997) 315 , Proceedings of the Third International Conference on Nuclear Physics at Storage Rings.

113. B. Franzke, Nuclear Instruments and Methods in Physics Research Section B: Beam Interactions with Materials and Atoms 24-25 (1987) 18 .

114. L. J. Mao, H. Zhao, X. D. Yang, J. Li, J. C. Yang, Y. J. Yuan, V. V. Parkhomchuk, V. B. Reva, X. M. Ma, T. L. Yan, M. T. Tang and J. W. Xia, Nuclear Instruments and Methods in Physics Research A $\mathbf{8 0 8}$ (Feb 2016) 29.

115. F. Lin, Y. S. Derbenev, D. Douglas, J. Guo, R. P. Johnson, G. Krafft, V. S. Morozov and Y. Zhang, arXiv e-prints (May 2016) arXiv:1605.02594, arXiv:1605.02594 [physics.acc-ph].

116. M. I. Bryzgunov, A. V. Bubley, A. P. Denisov, V. V. Parkhomchuk, V. M. Panasyuk, V. B. Reva, A. M. Batrakov, T. V. Bedareva, E. A. Bekhtenev, A. D. Goncharov, 
K. M. Gorchakov, I. A. Gusev, B. A. Dovzhenko, A. A. Zharikov, G. V. Karpov, V. V. Kolmogorov, M. N. Kondaurov, V. Y. Korchagin, N. S. Kremnev, V. A. Polukhin, A. A. Putmakov, D. V. Senkov, M. G. Fedotov and V. A. Chekavinskiy, Physics of Particles and Nuclei Letters 15 (Dec 2018) 758.

117. Y. A. Litvinov, Nucl. Phys. A 805 (Jun 2008) 260.

118. H. Geissel and Y. A. Litvinov, Nuclear Instruments and Methods in Physics Research Section B: Beam Interactions with Materials and Atoms 266 (2008) 4176 , Proceedings of the XVth International Conference on Electromagnetic Isotope Separators and Techniques Related to their Applications.

119. R. Knbel, M. Diwisch, F. Bosch, D. Boutin, L. Chen, C. Dimopoulou, A. Dolinskii, B. Franczak, B. Franzke, H. Geissel, M. Hausmann, C. Kozhuharov, J. Kurcewicz, S. Litvinov, G. Martinez-Pinedo, M. Mato, M. Mazzocco, G. Mnzenberg, S. Nakajima, C. Nociforo, F. Nolden, T. Ohtsubo, A. Ozawa, Z. Patyk, W. Pla, C. Scheidenberger, J. Stadlmann, M. Steck, B. Sun, T. Suzuki, P. Walker, H. Weick, M.-R. Wu, M. Winkler and T. Yamaguchi, Physics Letters B 754 (2016) 288.

120. R. Knöbel, M. Diwisch, H. Geissel, Y. A. Litvinov, Z. Patyk, W. R. Plaß, C. Scheidenberger, B. Sun, H. Weick, F. Bosch, D. Boutin, L. Chen, C. Dimopoulou, A. Dolinskii, B. Franczak, B. Franzke, M. Hausmann, C. Kozhuharov, J. Kurcewicz, S. A. Litvinov, M. Matoš, M. Mazzocco, G. Münzenberg, S. Nakajima, C. Nociforo, F. Nolden, T. Ohtsubo, A. Ozawa, J. Stadlmann, M. Steck, T. Suzuki, P. M. Walker, M. Winkler and T. Yamaguchi, The European Physical Journal A 52 (May 2016) 138.

121. F. Attallah, M. Hausmann, Y. Litvinov, T. Radon, J. Stadlmann, K. Beckert, F. Bosch, M. Falch, B. Franzke, H. Geissel, T. Kerscher, O. Klepper, H.-J. Kluge, C. Kozhuharov, K. E. G. Lbner, G. Mnzenberg, F. Nolden, Y. Novikov, Z. Patyk and H. Wollnik, Nuclear Physics A 701 (04 2002) 561.

122. B. Sun, R. Knbel, Y. Litvinov, H. Geissel, J. Meng, K. Beckert, F. Bosch, D. Boutin, C. Brandau, L. Chen, I. Cullen, C. Dimopoulou, B. Fabian, M. Hausmann, C. Kozhuharov, S. Litvinov, M. Mazzocco, F. Montes, G. Mnzenberg, A. Musumarra, S. Nakajima, C. Nociforo, F. Nolden, T. Ohtsubo, A. Ozawa, Z. Patyk, W. Pla, C. Scheidenberger, M. Steck, T. Suzuki, P. Walker, H. Weick, N. Winckler, M. Winkler and T. Yamaguchi, Nuclear Physics A 812 (2008) 1.

123. W. L. Zhan, H. S. Xu, G. Q. Xiao, J. W. Xia, H. W. Zhao and Y. J. Yuan, Nucl. Phys. A $\mathbf{8 3 4}$ (Mar 2010) 694.

124. Y. J. Yuan, J. C. Yang, J. W. Xia, P. Yuan, W. M. Qiao, D. Q. Gao, G. Q. Xiao, H. W. Zhao, H. S. Xu, M. T. Song, X. D. Yang, X. H. Cai, L. Z. Ma, X. T. Yang, K. T. Man, Y. He, Z. Z. Zhou, J. H. Zhang, Z. Xu, Y. Liu, R. S. Mao, W. Zhang, D. Z. Xie, L. T. Sun, Y. Q. Yang, D. Y. Yin, P. Li, J. Li, J. Shi, W. P. Chai, B. W. Wei and W. L. Zhan, Nuclear Instruments and Methods in Physics Research B $\mathbf{3 1 7}$ (Dec 2013) 214.

125. R. J. Chen, Y. J. Yuan, M. Wang, X. Xu, P. Shuai, Y. H. Zhang, X. L. Yan, Y. M. Xing, H. S. Xu, X. H. Zhou, Y. A. Litvinov, S. Litvinov, X. C. Chen, C. Y. Fu, W. W. Ge, Z. Ge, X. J. Hu, W. J. Huang, D. W. Liu, Q. Zeng and W. Zhang, Physica Scripta Volume T 166 (November 2015) 014044.

126. B.-H. Sun, J.-W. Zhao, X.-H. Zhang, L.-N. Sheng, Z.-Y. Sun, I. Tanihata, S. Terashima, Y. Zheng, L.-H. Zhu, L.-M. Duan, L.-C. He, R.-J. Hu, G.-S. Li, W.-J. Lin, W.-P. Lin, C.-Y. Liu, Z. Liu, C.-G. Lu, X.-W. Ma, L.-J. Mao, Y. Tian, F. Wang, M. Wang, S.-T. Wang, J.-W. Xia, X.-D. Xu, H.-S. Xu, Z.-G. Xu, J.-C. Yang, D.-Y. Yin, Y.-J. Yuan, W.-L. Zhan, Y.-H. Zhang and X.-H. Zhou, Science Bulletin 63 (2018) 78 .

127. X. Xing, W. Meng, Z. Yu-Hu, X. Hu-Shan, S. Peng, T. Xiao-Lin, Y. A. Litvi- 
nov, Z. Xiao-Hong, S. Bao-Hua, Y. You-Jin, X. Jia-Wen, Y. Jian-Cheng, K. Blaum, C. Rui-Jiu, C. Xiang-Cheng, F. Chao-Yi, G. Zhuang, H. Zheng-Guo, H. Wen-Jia, L. Da-Wei, L. Yi-Hua, M. Xin-Wen, M. Rui-Shi, T. Uesaka, X. Guo-Qing, X. YuanMing, T. Yamaguchi, Y. Yamaguchi, Z. Qi, Y. Xin-Liang, Z. Hong-Wei, Z. Tie-Cheng, Z. Wei and Z. Wen-Long, Chinese Physics C 39 (2015) 104001.

128. X. L. Tu, X. C. Chen, J. T. Zhang, P. Shuai, K. Yue, X. Xu, C. Y. Fu, Q. Zeng, X. Zhou, Y. M. Xing, J. X. Wu, R. S. Mao, L. J. Mao, K. H. Fang, Z. Y. Sun, M. Wang, J. C. Yang, Y. A. Litvinov, K. Blaum, Y. H. Zhang, Y. J. Yuan, X. W. Ma, X. H. Zhou and H. S. Xu, Phys. Rev. C 97 (Jan 2018) 014321.

129. X. Xu, M. Wang, Y.-H. Zhang, H.-S. Xu, P. Shuai, X.-L. Tu, A. L. Yuri, X.-H. Zhou, B.-H. Sun, Y.-J. Yuan, J.-W. Xia, J.-C. Yang, B. Klaus, R.-J. Chen, X.-C. Chen, C.-Y. Fu, Z. Ge, Z.-G. Hu, W.-J. Huang, D.-W. Liu, Y.-H. Lam, X.-W. Ma, R.-S. Mao, T. Uesaka, G.-Q. Xiao, Y.-M. Xing, T. Yamaguchi, Y. Yamaguchi, Q. Zeng, X.-L. Yan, H.-W. Zhao, T.-C. Zhao, W. Zhang and W.-L. Zhan, Chinese Physics $C$ 39 (October 2015) 106201, arXiv:1603.06404 [nucl-ex].

130. P. Zhang, X. Xu, P. Shuai, R. J. Chen, X. L. Yan, Y. H. Zhang, M. Wang, Y. A. Litvinov, K. Blaum, H. S. Xu, T. Bao, X. C. Chen, H. Chen, C. Y. Fu, J. J. He, S. Kubono, Y. H. Lam, D. W. Liu, R. S. Mao, X. W. Ma, M. Z. Sun, X. L. Tu, Y. M. Xing, J. C. Yang, Y. J. Yuan, Q. Zeng, X. Zhou, X. H. Zhou, W. L. Zhan, S. Litvinov, G. Audi, T. Uesaka, Y. Yamaguchi, T. Yamaguchi, A. Ozawa, B. H. Sun, Y. Sun and F. R. Xu, Physics Letters B 767 (Apr 2017) 20.

131. Y. H. Zhang, H. S. Xu, Y. A. Litvinov, X. L. Tu, X. L. Yan, S. Typel, K. Blaum, M. Wang, X. H. Zhou, Y. Sun, B. A. Brown, Y. J. Yuan, J. W. Xia, J. C. Yang, G. Audi, X. C. Chen, G. B. Jia, Z. G. Hu, X. W. Ma, R. S. Mao, B. Mei, P. Shuai, Z. Y. Sun, S. T. Wang, G. Q. Xiao, X. Xu, T. Yamaguchi, Y. Yamaguchi, Y. D. Zang, H. W. Zhao, T. C. Zhao, W. Zhang and W. L. Zhan, Phys. Rev. Lett. 109 (Sep 2012) 102501.

132. X. L. Yan, H. S. Xu, Y. A. Litvinov, Y. H. Zhang, H. Schatz, X. L. Tu, K. Blaum, X. H. Zhou, B. H. Sun, J. J. He, Y. Sun, M. Wang, Y. J. Yuan, J. W. Xia, J. C. Yang, G. Audi, G. B. Jia, Z. G. Hu, X. W. Ma, R. S. Mao, B. Mei, P. Shuai, Z. Y. Sun, S. T. Wang, G. Q. Xiao, X. XU, T. Yamaguchi, Y. Yamaguchi, Y. D. Zang, H. W. Zhao, T. C. Zhao, W. Zhang and W. L. Zhan, Astroph.J. 766 (Mar 2013) L8.

133. H. S. Xu, Y. H. Zhang and Y. A. Litvinov, International Journal of Mass Spectrometry 349-350 (2013) 162 , 100 years of Mass Spectrometry.

134. Y. M. Xing, K. A. Li, Y. H. Zhang, X. H. Zhou, M. Wang, Y. A. Litvinov, K. Blaum, S. Wanajo, S. Kubono, G. Martínez-Pinedo, A. Sieverding, R. J. Chen, P. Shuai, C. Y. Fu, X. L. Yan, W. J. Huang, X. Xu, X. D. Tang, H. S. Xu, T. Bao, X. C. Chen, B. S. Gao, J. J. He, Y. H. Lam, H. F. Li, J. H. Liu, X. W. Ma, R. S. Mao, M. Si, M. Z. Sun, X. L. Tu, Q. Wang, J. C. Yang, Y. J. Yuan, Q. Zeng, P. Zhang, X. Zhou, W. L. Zhan, S. Litvinov, G. Audi, T. Uesaka, Y. Yamaguchi, T. Yamaguchi, A. Ozawa, C. Fröhlich, T. Rauscher, F. K. Thielemann, B. H. Sun, Y. Sun, A. C. Dai and F. R. Xu, Physics Letters B 781 (Jun 2018) 358, arXiv: 1804.02309 [nucl-ex].

135. X. L. Tu, H. S. Xu, M. Wang, Y. H. Zhang, Y. A. Litvinov, Y. Sun, H. Schatz, X. H. Zhou, Y. J. Yuan, J. W. Xia, G. Audi, K. Blaum, C. M. Du, P. Geng, Z. G. Hu, W. X. Huang, S. L. Jin, L. X. Liu, Y. Liu, X. Ma, R. S. Mao, B. Mei, P. Shuai, Z. Y. Sun, H. Suzuki, S. W. Tang, J. S. Wang, S. T. Wang, G. Q. Xiao, X. Xu, T. Yamaguchi, Y. Yamaguchi, X. L. Yan, J. C. Yang, R. P. Ye, Y. D. Zang, H. W. Zhao, T. C. Zhao, X. Y. Zhang and W. L. Zhan, Phys. Rev. Lett. 106 (Mar 2011) 112501. 
136. P. Shuai, H. S. Xu, X. L. Tu, Y. H. Zhang, B. H. Sun, Y. A. Litvinov, X. L. Yan, K. Blaum, M. Wang, X. H. Zhou, J. J. He, Y. Sun, K. Kaneko, Y. J. Yuan, J. W. Xia, J. C. Yang, G. Audi, X. C. Chen, G. B. Jia, Z. G. Hu, X. W. Ma, R. S. Mao, B. Mei, Z. Y. Sun, S. T. Wang, G. Q. Xiao, X. Xu, T. Yamaguchi, Y. Yamaguchi, Y. D. Zang, H. W. Zhao, T. C. Zhao, W. Zhang and W. L. Zhan, arXiv e-prints (Apr 2014) arXiv:1404.1187, arXiv:1404.1187 [nucl-ex].

137. Z. Sun, W.-L. Zhan, Z.-Y. Guo, G. Xiao and J.-X. Li, Nuclear Instruments and Methods in Physics Research Section A: Accelerators, Spectrometers, Detectors and Associated Equipment 503 (2003) 496 .

138. B. Mei, X. Tu, M. Wang, H. Xu, R. Mao, Z. Hu, X. Ma, Y. Yuan, X. Zhang, P. Geng, P. Shuai, Y. Zang, S. Tang, P. Ma, W. Lu, X. Yan, J. Xia, G. Xiao, Z. Guo, H. Zhang and K. Yue, Nuclear Instruments and Methods in Physics Research A 624 (Dec 2010) 109.

139. X. Tu, M. Wang, Y. Litvinov, Y. Zhang, H. Xu, Z. Sun, G. Audi, K. Blaum, C. Du, W. Huang, Z. Hu, P. Geng, S. Jin, L. Liu, Y. Liu, B. Mei, R. Mao, X. Ma, H. Suzuki, P. Shuai, Y. Sun, S. Tang, J. Wang, S. Wang, G. Xiao, X. Xu, J. Xia, J. Yang, R. Ye, T. Yamaguchi, X. Yan, Y. Yuan, Y. Yamaguchi, Y. Zang, H. Zhao, T. Zhao, X. Zhang, X. Zhou and W. Zhan, Nuclear Instruments and Methods in Physics Research Section A: Accelerators, Spectrometers, Detectors and Associated Equipment 654 (2011) 213.

140. T. Dickel, W. R. Plaß, J. Lang, J. Ebert, H. Geissel, E. Haettner, C. Jesch, W. Lippert, M. Petrick, C. Scheidenberger and M. I. Yavor, Nuclear Instruments and Methods in Physics Research B $\mathbf{3 1 7}$ (Dec 2013) 779.

141. P. Schury, M. Wada, Y. Ito, D. Kaji, F. Arai, M. MacCormick, I. Murray, H. Haba, S. Jeong, S. Kimura, H. Koura, H. Miyatake, K. Morimoto, K. Morita, A. Ozawa, M. Rosenbusch, M. Reponen, P.-A. Söderström, A. Takamine, T. Tanaka and H. Wollnik, Phys. Rev. C 95 (Jan 2017) 011305.

142. P. Schury, Y. Ito, M. Rosenbusch, H. Miyatake, M. Wada and H. Wollnik, International Journal of Mass Spectrometry 433 (Oct 2018) 40, arXiv:1805.07023 [physics.ins-det].

143. P. Schury, M. Wada, Y. Ito, S. Naimi, T. Sonoda, H. Mita, A. Takamine, K. Okada, H. Wollnik, S. Chon, H. Haba, D. Kaji, H. Koura, H. Miyatake, K. Morimoto, K. Morita and A. Ozawa, Nuclear Instruments and Methods in Physics Research Section B: Beam Interactions with Materials and Atoms 317 (2013) 537 , XVIth International Conference on ElectroMagnetic Isotope Separators and Techniques Related to their Applications, December 27, 2012 at Matsue, Japan.

144. P. Chauveau, P. Delahaye, G. De France, S. El Abir, J. Lory, Y. Merrer, M. Rosenbusch, L. Schweikhard and R. N. Wolf, Nuclear Instruments and Methods in Physics Research B $\mathbf{3 7 6}$ (Jun 2016) 211.

145. M. Brodeur, J. Kelly, B. Liu and B. Schultz, Off-line commissioning of the University of Notre Dame Multi-Reflection Time-of-Flight mass spectrograph, in APS Division of Nuclear Physics Meeting Abstracts, (Sep 2017), p. FD.002.

146. T. Y. Hirsh, N. Paul, M. Burkey, A. Aprahamian, F. Buchinger, S. Caldwell, J. A. Clark, A. F. Levand, L. L. Ying, S. T. Marley, G. E. Morgan, A. Nystrom, R. Orford, A. P. Galván, J. Rohrer, G. Savard, K. S. Sharma and K. Siegl, Nuclear Instruments and Methods in Physics Research B 376 (Jun 2016) 229.

147. P. Schury, M. Wada, Y. Ito, F. Arai, S. Naimi, T. Sonoda, H. Wollnik, V. Shchepunov, C. Smorra and C. Yuan, Nuclear Instruments and Methods in Physics Research Section B: Beam Interactions with Materials and Atoms 335 (2014) 39 .

148. C. Jesch, T. Dickel, W. R. Plaß, D. Short, S. Ayet San Andres, J. Dilling, H. Geissel, 
F. Greiner, J. Lang, K. G. Leach, W. Lippert, C. Scheidenberger and M. I. Yavor, Hyperfine Interactions 235 (Nov 2015) 97.

149. W. R. Plaß, T. Dickel and C. Scheidenberger, International Journal of Mass Spectrometry 349-350 (Sep 2013) 134.

150. D. Lunney, C. Monsanglant, G. Audi, G. Bollen, C. Borcea, H. Doubre, C. Gaulard, S. Henry, M. deSaintSimon, C. Thibault, C. Toader and N. Vieira, Hyperfine Interactions 132 (Jan 2001) 297.

151. M. de Saint Simon, C. Thibault, G. Audi, A. Coc, H. Doubre, M. Jacotin, J.-F. Kepinski, R. L. Gac, G. L. Scornet, D. Lunney, F. Touchard and G. Lebe, Physica Scripta 1995 (1995) 406

152. C. Gaulard, G. Audi, C. Bachelet, D. Lunney, M. de Saint Simon, C. Thibault and N. Vieira, Nuclear Physics A 766 (2006) 52 .

153. C. Bachelet, G. Audi, C. Gaulard, C. Guénaut, F. Herfurth, D. Lunney, M. de Saint Simon and C. Thibault, Phys. Rev. Lett. 100 (May 2008) 182501.

154. C. Gaulard, C. Bachelet, G. Audi, C. Gunaut, D. Lunney, M. de Saint Simon, M. Sewtz and C. Thibault, Nuclear Physics A 826 (2009) 1.

155. Nuclear masses.

156. National nuclear data center.

157. G. Audi, O. Bersillon, J. Blachot and A. Wapstra, Nuclear Physics A 729 (2003) 3 , The 2003 NUBASE and Atomic Mass Evaluations.

158. A. Wapstra, G. Audi and C. Thibault, Nuclear Physics A 729 (2003) 129 , The 2003 NUBASE and Atomic Mass Evaluations.

159. G. Audi, A. Wapstra and C. Thibault, Nuclear Physics A 729 (2003) 337, The 2003 NUBASE and Atomic Mass Evaluations.

160. G. Audi, F. Kondev, M. Wang, W. Wang and S. Naimi, Chinese Physics C 41 (2017) 03001 .

161. The atomic mass data center.

162. Nuclear data services: Atomic mass data center.

163. B. Pfeiffer, K. Venkataramaniah, U. Czok and C. Scheidenberger, Atomic Data and Nuclear Data Tables 100 (2014) 403.

164. G. Audi, K. Blaum, M. Block, G. Bollen, S. Goriely, J. Hardy, F. Herfurth, F. Kondev, H.-J. Kluge, D. Lunney, J. Pearson, G. Savard, K. Sharma, M. Wang and Y. Zhang, Atomic Data and Nuclear Data Tables 103-104 (2015) 1.

165. Surman, Rebecca and Mumpower, Matthew, EPJ Web Conf. 178 (2018) 04002.

166. O. Kester, P. Spiller and H. Stoecker, Fair project at gsi, in Challenges and Goals for Accelerators in the XXI Century, (2018), pp. 611-622.

167. W. Barth, A. Adonin, C. E. Düllmann, M. Heilmann, R. Hollinger, E. Jäger, O. Kester, J. Khuyagbaatar, J. Krier, E. Plechov, P. Scharrer, W. Vinzenz, H. Vormann, A. Yakushev and S. Yaramyshev, Phys. Rev. Accel. Beams 20 (May 2017) 050101 .

168. G. Mnzenberg and H. Geissel, Journal of Physics: Conference Series 413 (Feb 2013) 012006 .

169. J. Gerl, The nustar project at gsi and fair, in Exotic Nuclei, (2013), pp. 495-509.

170. C. Dimopoulou, K. Beckert, P. Beller, A. Dolinskii, U. Laier, F. Nolden, G. Schreiber, M. Steck and J. Yang, Phys. Rev. ST Accel. Beams 10 (Feb 2007) 020101.

171. S. Jeong, P. Papakonstantinou, H. Ishiyama and Y. Kim, Journal of the Korean Physical Society 73 (Aug 2018) 516.

172. J. Yang, J. Xia, G. Xiao, H. Xu, H. Zhao, X. Zhou, X. Ma, Y. He, L. Ma, D. Gao, J. Meng, Z. Xu, R. Mao, W. Zhang, Y. Wang, L. Sun, Y. Yuan, P. Yuan, W. Zhan, J. Shi, W. Chai, D. Yin, P. Li, J. Li, L. Mao, J. Zhang and L. Sheng, Nuclear Instru- 
ments and Methods in Physics Research Section B: Beam Interactions with Materials and Atoms 317 (2013) 263 , XVIth International Conference on ElectroMagnetic Isotope Separators and Techniques Related to their Applications, December 27, 2012 at Matsue, Japan.

173. B. Wu, J. Yang, J. Xia, X. Yan, X. Hu, L. Mao, L. Sheng, J. Wu, D. Yin, W. Chai, G. Shen, W. Ge, G. Wang, H. Zhao, S. Ruan, X. Ma, M. Wang, S. Litvinov, W. Wen, X. Chen, R. Chen, M. Tang, W. Wu, C. Luo, T. Zhao, C. Shi, X. Fu, J. Liu and L. Liang, Nuclear Instruments and Methods in Physics Research Section A: Accelerators, Spectrometers, Detectors and Associated Equipment 881 (2018) 27.

174. S. Michimasa, S. Ota, M. Dozono and M. T. et al., Sharaq spectrometer: Highresolution spectroscopy using exotic beams and reactions, in The 26th International Nuclear Physics Conference, ed. P. of Science (2017). 